\title{
Combining non-cointegration tests
}

Citation for published version (APA):

Bayer, C., \& Hanck, C. H. (2009). Combining non-cointegration tests. METEOR, Maastricht University School of Business and Economics. METEOR Research Memorandum No. 012 https://doi.org/10.26481/umamet.2009012

Document status and date:

Published: 01/01/2009

DOI:

10.26481/umamet.2009012

Document Version:

Publisher's PDF, also known as Version of record

\section{Please check the document version of this publication:}

- A submitted manuscript is the version of the article upon submission and before peer-review. There can be important differences between the submitted version and the official published version of record.

People interested in the research are advised to contact the author for the final version of the publication, or visit the DOI to the publisher's website.

- The final author version and the galley proof are versions of the publication after peer review.

- The final published version features the final layout of the paper including the volume, issue and page numbers.

Link to publication

\footnotetext{
General rights rights.

- You may freely distribute the URL identifying the publication in the public portal. please follow below link for the End User Agreement:

www.umlib.nl/taverne-license

Take down policy

If you believe that this document breaches copyright please contact us at:

repository@maastrichtuniversity.nl

providing details and we will investigate your claim.
}

Copyright and moral rights for the publications made accessible in the public portal are retained by the authors and/or other copyright owners and it is a condition of accessing publications that users recognise and abide by the legal requirements associated with these

- Users may download and print one copy of any publication from the public portal for the purpose of private study or research.

- You may not further distribute the material or use it for any profit-making activity or commercial gain

If the publication is distributed under the terms of Article $25 \mathrm{fa}$ of the Dutch Copyright Act, indicated by the "Taverne" license above, 


\section{Maastricht University}

Christian Bayer, Christoph Hanck

Combining Non-Cointegration Tests

$\mathrm{RM} / 09 / 012$

(RM/08/014 -revised-)

\section{METEOR}

Faculty of Economics and Business Administration Maastricht Research School of Economics

of Technology and Organization

\section{P.O. Box 616}

NL - 6200 MD Maastricht

The Netherlands 


\title{
Combining Non-Cointegration Tests*
}

\author{
Christian Bayer ${ }^{\dagger}$ \\ Christoph Hanck ${ }^{\ddagger}$ \\ Università Commerciale L. Bocconi Universiteit Maastricht
}

First Version: May 2008

March 10, 2009

\section{Preliminary.}

\begin{abstract}
The local asymptotic power of many popular non-cointegration tests has recently been shown to depend on a certain nuisance parameter. Depending on the value of that parameter, different tests perform best. This paper suggests combination procedures with the aim of providing meta tests that maintain high power across the range of the nuisance parameter. The local asymptotic power of the new meta tests is in general almost as high as that of the more powerful of the underlying tests. When the underlying tests have similar power, the meta tests are even more powerful than the best underlying test. At the same time, our new meta tests avoid the arbitrary decision which test to use if single test results conflict. Moreover it avoids the size distortion inherent in separately applying multiple tests for cointegration to the same data set. We apply our tests to 159 data sets from published cointegration studies. There, in one third of all cases single tests give conflicting results whereas our meta tests provide an unambiguous test decision.
\end{abstract}

Keywords: Cointegration, Meta Test, Multiple Testing

JEL-Codes: C12, C22

${ }^{*}$ This is a substantially extended version of a manuscript that previously circulated under the title 'Is Double Trouble? How to Combine Cointegration Tests'. Part of the research was carried out when the authors were at Technische Universität Dortmund with support by DFG under Sonderforschungsbereich 475. We are grateful to Carsten Burhop, Carlo Favero, Alain Hecq, Walter Krämer and Franz Palm as well as conference and seminar participants in Maastricht, Neuchâtel and Bonn for valuable comments and suggestions.

${ }^{\dagger}$ IGIER, Via Salasco 5, 20136 Milano, Italy. Tel.: +39 (0)2 5836 3386. email: christian.bayer@unibocconi.it.

${ }^{\ddagger}$ Department of Quantitative Economics, Tongersestraat 53, 6211LM Maastricht, Netherlands. Tel.: +31 (0)43 3883815. email: c.hanck@ke.unimaas.nl. 


\section{Introduction}

Testing for cointegration has become one of the standard tools in applied economic research. Various tests have been suggested for this purpose, most of which are implemented in standard econometric packages and hence are easily available nowadays. Well-known examples include the residual-based test of Engle and Granger (1987), or the system-based tests of Johansen (1988). Error-Correction-based tests have been suggested by Boswijk (1994) and Banerjee et al. (1998), while Breitung (2001) covers the nonlinear case - to name just a few. This regularly forces the applied researcher to select from the test decisions of the various applicable procedures. This choice is difficult because, as discussed in e.g. Elliott et al. (2005), there exists no uniformly most powerful test, even asymptotically. Often one test rejects the null hypothesis whereas another test does not, making it unclear how to interpret test outcomes then. More generally speaking, the $p$-values of different tests are typically not perfectly correlated (Gregory et al., 2004).

This imperfect correlation rules out relying, for example, on the test that achieves the smallest $p$-value. Such strategy will not control the probability of rejecting a true null hypothesis at some chosen level $\alpha$ because it ignores the multiple testing nature of the problem. Concretely, using the test with the smallest $p$-value will lead to an oversized test.

The imperfect correlation of different test statistics reflects that the tests are not equivalent, focussing on different statistical characterizations of non-cointegration. This also has implications for their behavior under the alternative. Specifically, Pesavento (2004) shows that the relative power of cointegration tests depends crucially on the squared long-run correlations of error terms driving the variables of the analyzed system. That is, the power ranking of the tests varies by the value of that unknown nuisance parameter.

This suggests that suitable combinations of non-cointegration tests potentially yield a more robust power performance, and possibly even power gains, relative to applying only a single test. Based on the above-mentioned single cointegration tests, the present paper develops such combination tests. In particular, we propose to combine test statistics in the spirit of Fisher's (1932) famous test. We derive the asymptotic null distribution of our Fisher-type combination test for correlated cointegration test statistics and its local asymptotic power, exploiting Pesavento's (2004) results. Besides successfully tackling the above-mentioned multiple testing problem inherent in combining different test statistics, the combined test indeed enjoys a robust power performance over the range of the squared long-run error correlation. Moreover, we explore a number of alternative combination procedures. For example, Harvey et al. (2009) propose a Union-of-Rejections procedure to robustify unit root tests against uncertainty over the initial condition. We generalize their idea and apply the generalized Union-of-Rejections approach to the present testing problem.

Our Fisher-type test turns out to perform very well. It follows closely the power envelope of the underlying single tests, and even exceeds it when the single tests have similar power. In contrast, the Union-of-Rejections procedure is most useful when the underlying tests have strongly different power, in that its power is always close to that of the better underlying test. 
Of course, the asymptotic distributions derived here are, as usual, only approximations to the generally analytically intractable finite-sample distributions. Those may or may not be accurate. We therefore additionally propose bootstrap analogs of our combination tests. Specifically, we build on Swensen's (2006) recent bootstrap scheme for cointegrated vector autoregressions.

We conduct extensive finite-sample experiments of the performance our asymptotic and bootstrap combination tests. The local asymptotic results correctly predict the finite-sample performance. Both the asymptotic and the bootstrap versions successfully control the level $\alpha$ of the test and are at the same time powerful. The bootstrap versions appear to converge to the nominal size somewhat more quickly.

We point out that the above multiple testing problem is pervasive in empirical work and not restricted to testing for cointegration. The meta testing solution developed here is rather general and could hence be adopted to other testing problems for which several (imperfectly correlated) tests have been developed. Examples include testing for unit roots or heteroscedasticity.

To check the practical relevance of our proposed tests, we revisit the set of published studies that Gregory et al. (2004) examined for 'mixed signals' among cointegration tests, i.e. conflicting test results. Among other things we find that in one third of all cases single tests give conflicting results. In these cases our meta tests are particularly useful. They provide an unambiguous test decision and therefore are a solution to the 'mixed signals' problem.

The remainder of this paper is organized as follows: Section 2 provides the setup for the noncointegration tests. Section 3 derives our combination tests. Section 4 presents local asymptotic power results. Section 5 is devoted to the bootstrap analogs. Section 6 reports Monte Carlo results. Section 7 provides the empirical application. Section 8 concludes. An appendix reports additional results.

The notation is standard. Weak convergence, convergence in probability and in distribution are denoted by $\Rightarrow, \rightarrow_{p}$ and $\rightarrow_{d}$. Limits of integration are 0 and $1, \int=\int_{0}^{1}$, unless specified otherwise. $[a]$ is the integer part of $a$. Vectors and matrices are given in boldface. Integrals such as $\int_{0}^{1} \boldsymbol{W}(s) \boldsymbol{W}(s)^{\prime} \mathrm{d} s$ will often be written as $\int \boldsymbol{W} \boldsymbol{W}^{\prime}$. When $a$ defines $b$, we write $b:=a$ or $a=: b$.

\section{Setup}

\subsection{Model}

Let $\boldsymbol{z}_{t}:=\left(z_{1 t}, \ldots, z_{K t}\right)^{\prime} \in \mathbb{R}^{K}$ be a vector of stochastic variables integrated of order one, $I(1)$. Partition $\boldsymbol{z}_{t}=\left(\boldsymbol{x}_{t}^{\prime}, y_{t}\right)^{\prime}$. Suppose we observe $\boldsymbol{z}_{0}, \ldots, \boldsymbol{z}_{T}$. We work with the model studied by Pesavento (2004):

$$
\begin{aligned}
\Delta \boldsymbol{x}_{t} & =\boldsymbol{\tau}_{1}+\boldsymbol{v}_{1 t} \\
y_{t} & =\left(\mu_{2}-\boldsymbol{\theta}^{\prime} \boldsymbol{\mu}_{1}\right)+\left(\tau_{2}-\boldsymbol{\theta}^{\prime} \boldsymbol{\tau}_{1}\right) t+\boldsymbol{\theta}^{\prime} \boldsymbol{x}_{t}+u_{t} \\
u_{t} & =\rho u_{t-1}+v_{2 t}
\end{aligned}
$$


We make the following assumption on the error vector $\boldsymbol{v}_{t}:=\left(\boldsymbol{v}_{1 t}^{\prime}, v_{2 t}\right)^{\prime}$ from eqs. (1a) and (1c). Assumption 1. $\left\{\boldsymbol{v}_{t}\right\}$ satisfies a Functional Central Limit Theorem, i.e. $T^{-1 / 2} \sum_{t=1}^{[\lambda T]} \boldsymbol{v}_{t} \Rightarrow \boldsymbol{\Omega}^{1 / 2} \boldsymbol{W}(\lambda)$, with $\boldsymbol{\Omega}$ the long-run covariance matrix of $\boldsymbol{v}_{t}$.

Equation (1a) defines the dynamics of the regressors, while eqs. (1b) and (1c) describe the (single potential) cointegrating relationship. ${ }^{1}$ The coefficients $\boldsymbol{\mu}:=\left(\boldsymbol{\mu}_{1}^{\prime}, \mu_{2}\right)^{\prime}$ and $\boldsymbol{\tau}:=\left(\boldsymbol{\tau}_{1}^{\prime}, \tau_{2}\right)^{\prime}$ determine the specification of the deterministic components of the model, see Definition 1 below and Pesavento (2004) for details. The vector $\boldsymbol{z}_{t}$ is said to be cointegrated if there exists at least one $\tilde{\boldsymbol{\theta}} \in \mathbb{R}^{K}, \tilde{\boldsymbol{\theta}}:=\left(-\boldsymbol{\theta}^{\prime}, 1\right)^{\prime}, \boldsymbol{\theta} \neq \mathbf{0}$, such that the stochastic part of $\tilde{\boldsymbol{\theta}}^{\prime} \boldsymbol{z}_{t}$ is a stationary $I(0)$ process. In terms of (1), cointegration therefore obtains if $|\rho|<1$. We test the null hypothesis

$\mathcal{H}_{0}$ : There exists no cointegrating relationship among the variables in $\boldsymbol{z}_{t}$. against the alternative hypothesis

$\mathcal{H}_{1}$ : There exists a $\tilde{\boldsymbol{\theta}} \neq \mathbf{0}$ such that the stochastic part of $\tilde{\boldsymbol{\theta}}^{\prime} \boldsymbol{z}_{t}$ is $I(0)$.

The literature has suggested various tests to discriminate between $\mathcal{H}_{0}$ and $\mathcal{H}_{1}$. We consider the residual-based test of Engle and Granger (1987), a system-based test of Johansen (1988), as well as the error-correction-based tests of Boswijk (1994) and Banerjee et al. (1998). Pesavento (2004) derives the local asymptotic power of these tests. She shows that, under (1), their power only depends on the local-to-unity parameter $c:=T(\rho-1)$ and the squared correlations of the elements of $\boldsymbol{v}_{1 t}$ with $v_{2 t}$. More precisely, partition $\boldsymbol{\Omega}$ conformably with $\left(\boldsymbol{x}_{t}^{\prime}, y_{t}\right)^{\prime}$,

$$
\boldsymbol{\Omega}=\left(\begin{array}{ll}
\boldsymbol{\Omega}_{11} & \boldsymbol{\omega}_{12} \\
\boldsymbol{\omega}_{12}^{\prime} & \omega_{22}
\end{array}\right)
$$

We define the squared correlation as $R^{2}:=\boldsymbol{\delta}^{\prime} \boldsymbol{\delta}$, where $\boldsymbol{\delta}:=\boldsymbol{\Omega}_{11}^{-1 / 2} \boldsymbol{\omega}_{12} \omega_{22}^{-1 / 2}$. This requires $\boldsymbol{\Omega}_{11}$ to be invertible, which is implied by

Assumption 2. There are no cointegrating relationships among the variables in $\boldsymbol{x}_{t}$.

$R^{2}=0$ corresponds to Kremers et al.'s (1992) 'common factor restriction'. Moreover, we partition $\boldsymbol{W}:=\left(\boldsymbol{W}_{1}^{\prime}, W_{2}\right)^{\prime}$. Define the Ornstein-Uhlenbeck process $J_{12 c}(\lambda):=W_{12}(\lambda)+c \int_{0}^{\lambda} \mathrm{e}^{(\lambda-s) c} W_{12}(s) \mathrm{d} s$, with $W_{12}:=\overline{\boldsymbol{\delta}}^{\prime} \boldsymbol{W}_{1}+W_{2}$, where $\overline{\boldsymbol{\delta}}^{\prime} \overline{\boldsymbol{\delta}}=\frac{R^{2}}{1-R^{2}}$. Furthermore, we distinguish the following cases.

Definition 1. Depending on the assumptions made about the deterministic components, we have

(i) $\boldsymbol{W}^{d}(\lambda):=\boldsymbol{W}(\lambda)$ and $J_{12 c}^{d}(\lambda)=J_{12 c}(\lambda)$ if $\mu_{2}-\boldsymbol{\theta}^{\prime} \boldsymbol{\mu}_{1}=0, \boldsymbol{\tau}=\mathbf{0}$ and no deterministic terms are included in the regressions. We refer to this as case $(i)$.

(ii) $\boldsymbol{W}^{d}(\lambda):=\boldsymbol{W}(\lambda)-\int \boldsymbol{W}(s) \mathrm{d} s$ and $J_{12 c}^{d}(\lambda)=J_{12 c}(\lambda)-\int J_{12 c}(s) \mathrm{d} s$ if $\boldsymbol{\tau}=\mathbf{0}$ and a constant is included in the regressions. We refer to this as case (ii).

(iii) $\boldsymbol{W}^{d}(\lambda):=\boldsymbol{W}(\lambda)-(4-6 \lambda) \int \boldsymbol{W}(s) \mathrm{d} s-(12 \lambda-6) \int s \boldsymbol{W}(s) \mathrm{d} s$ and $J_{12 c}^{d}(\lambda)=J_{12 c}(\lambda)-(4-$ $6 \lambda) \int J_{12 c}(s) \mathrm{d} s-(12 \lambda-6) \int s \boldsymbol{W}(s) \mathrm{d} s$ if there are no restrictions and a constant and trend are included in the regressions. We refer to this as case (iii).

Also, $\boldsymbol{W}_{c}^{d}:=\left(\boldsymbol{W}_{1}^{d^{\prime}}(\lambda), J_{12 c}^{d}(\lambda)\right)^{\prime}$ and $\boldsymbol{A}_{c}^{d}:=\int \boldsymbol{W}_{c}^{d} \boldsymbol{W}_{c}^{d^{\prime}}$.

\footnotetext{
${ }^{1}$ Pesavento (2004) shows that (1) does not generally impose weak exogeneity.
} 


\subsection{Single Cointegration Tests}

Engle and Granger (1987)

The Engle-Granger test tests $\mathcal{H}_{0}$ against the alternative of at least one cointegrating relationship. One computes the $t$-statistic $t_{\gamma}^{\mathrm{ADF}}$ on $\gamma$ in the OLS regression

$$
\Delta \hat{u}_{t}=\gamma \hat{u}_{t-1}+\sum_{p=1}^{P-1} \nu_{p} \Delta \hat{u}_{t-p}+\epsilon_{t} .
$$

Here, $\hat{u}_{t}$ is the usual residual from a first stage OLS regression of $y_{t}$ on $\boldsymbol{x}_{t}$ (and appropriate deterministic terms). The sum $\sum_{p=1}^{P-1} \nu_{p} \Delta \hat{u}_{t-p}$ captures residual serial correlation. ${ }^{2}$ Proposition 1 summarizes the local asymptotic distribution derived by Pesavento (2004).

Proposition 1. With the terms as in Definition 1, we have

$$
\begin{aligned}
& t_{\gamma}^{\mathrm{ADF}} \Rightarrow c \frac{\left(\boldsymbol{\eta}_{c}^{d^{\prime}} \boldsymbol{A}_{c}^{d} \boldsymbol{\eta}_{c}^{d}\right)^{1 / 2}}{\left(\boldsymbol{\eta}_{c}^{d^{\prime}} \boldsymbol{D} \boldsymbol{\eta}_{c}^{d}\right)^{1 / 2}}+\frac{\boldsymbol{\eta}_{c}^{d^{\prime}} \int \boldsymbol{W}_{c}^{d} \mathrm{~d} \widetilde{\boldsymbol{W}^{\prime}} \boldsymbol{\eta}_{c}^{d}}{\left(\boldsymbol{\eta}_{c}^{d^{\prime}} \boldsymbol{A}_{c}^{d} \boldsymbol{\eta}_{c}^{d}\right)^{1 / 2}\left(\boldsymbol{\eta}_{c}^{d^{\prime}} \boldsymbol{D} \boldsymbol{\eta}_{c}^{d}\right)^{1 / 2}} \\
& \text { where } \boldsymbol{\eta}_{c}^{d}:=\left[-\left(\int \boldsymbol{W}_{1}^{d^{\prime}} J_{12 c}^{d}\right)\left(\int \boldsymbol{W}_{1}^{d} \boldsymbol{W}_{1}^{d^{\prime}}\right)^{-1}, 1\right]^{\prime} \text {, } \\
& \widetilde{\boldsymbol{W}}(\lambda):=\left(\boldsymbol{W}_{1}^{\prime}(\lambda), \quad W_{12}(\lambda)\right)^{\prime}, \\
& D:=\left(\begin{array}{cc}
\boldsymbol{I} & \overline{\boldsymbol{\delta}} \\
\overline{\boldsymbol{\delta}}^{\prime} & 1+\overline{\boldsymbol{\delta}}^{\prime} \overline{\boldsymbol{\delta}}
\end{array}\right)
\end{aligned}
$$

Johansen (1988)

The system-based tests of Johansen (1988) test for $h$ cointegrating relationships. In view of $\mathcal{H}_{0}$, we consider $h=0$ throughout. One estimates the Vector Error Correction Model (VECM)

$$
\Delta \boldsymbol{z}_{t}=\boldsymbol{\Pi} \boldsymbol{z}_{t-1}+\sum_{p=1}^{P-1} \boldsymbol{\Gamma}_{p} \Delta \boldsymbol{z}_{t-p}+\boldsymbol{d}_{t}+\varepsilon_{t}
$$

with $\boldsymbol{d}_{t}$ appropriate deterministic terms. We employ the $\lambda_{\max }$ test with test statistic

$$
\lambda_{\max }(h)=-T \ln \left(1-\hat{\pi}_{1}\right) .
$$

Here, $\hat{\pi}_{1}$ denotes the largest solution to $\left|\pi \boldsymbol{S}_{11}-\boldsymbol{S}_{10} \boldsymbol{S}_{00}^{-1} \boldsymbol{S}_{01}\right|=0$ (in Johansen's (1995) notation). Again from Pesavento (2004), we obtain

Proposition 2. With the terms as in Definition 1 and $\boldsymbol{G}_{c}:=\int \boldsymbol{W}_{c}^{d} J_{12 c}\left(\mathbf{0}^{\prime}, c\right)$, we have

$$
\begin{aligned}
\lambda_{\max } \Rightarrow \max \operatorname{eig}\{ & \left(\boldsymbol{A}_{c}^{d}\right)^{-1}\left[\int \boldsymbol{W}_{c}^{d} \mathrm{~d} \boldsymbol{W}^{\prime} \int \mathrm{d} \boldsymbol{W} \boldsymbol{W}_{c}^{d^{\prime}}+\int \boldsymbol{W}_{c}^{d} \mathrm{~d} \boldsymbol{W}^{\prime} \boldsymbol{G}_{c}^{\prime}\right. \\
& \left.\left.+\boldsymbol{G}_{c}\left(\int \boldsymbol{W}_{c}^{d} \mathrm{~d} \boldsymbol{W}^{\prime}\right)^{\prime}+\boldsymbol{G}_{c} \boldsymbol{G}_{c}^{\prime}\right]\right\}
\end{aligned}
$$

\footnotetext{
${ }^{2}$ One could also control for serial correlation by the semiparametric approach of Phillips and Ouliaris (1990).
} 
Boswijk (1994) and Banerjee et al. (1998)

Banerjee et al. (1998) and Boswijk (1994) work with the conditional error correction representation of model (1). The equation to be estimated (by OLS) becomes

$$
\Delta y_{t}=d_{t}+\boldsymbol{\pi}_{0 x}^{\prime} \Delta \boldsymbol{x}_{t}+\varphi_{0} y_{t-1}+\boldsymbol{\varphi}_{1}^{\prime} \boldsymbol{x}_{t-1}+\sum_{p=1}^{P}\left(\boldsymbol{\pi}_{p x}^{\prime} \Delta \boldsymbol{x}_{t-p}+\pi_{p y} \Delta y_{t-p}\right)+\epsilon_{t},
$$

with $P$ chosen such that $\epsilon_{t}$ is approximately white noise. Banerjee et al.'s test statistic $t_{\gamma}^{\mathrm{ECR}}$ is the $t$-ratio for $\mathcal{H}_{0}: \varphi_{0}=0$, whereas Boswijk's $\hat{F}$ is the Wald statistic for $\mathcal{H}_{0}:\left(\varphi_{0}, \varphi_{1}^{\prime}\right)^{\prime}=\mathbf{0}$.

Proposition 3 (Pesavento, 2004). With the terms as in Definition 1, we have

$$
\begin{aligned}
\hat{F} \Rightarrow c^{2} \int J_{12 c}^{d 2}+2 c \int J_{12 c}^{d} \mathrm{~d} W_{2}+\int \boldsymbol{W}_{c}^{d^{\prime}} \mathrm{d} W_{2}\left(\boldsymbol{A}_{c}^{d}\right)^{-1} \int \boldsymbol{W}_{c}^{d} \mathrm{~d} W_{2} \\
t_{\gamma}^{\mathrm{ECR}} \Rightarrow c\left[\int J_{12 c}^{d 2}-\int \boldsymbol{W}_{1}^{d^{\prime}} J_{12 c}^{d}\left(\int \boldsymbol{W}_{1}^{d} \boldsymbol{W}_{1}^{d^{\prime}}\right)^{-1} \int \boldsymbol{W}_{1}^{d} J_{12 c}^{d}\right]^{1 / 2} \\
+\frac{\int J_{12 c}^{d} \mathrm{~d} W_{2}-\int \boldsymbol{W}_{1}^{d^{\prime}} J_{12 c}^{d}\left(\int \boldsymbol{W}_{1}^{d} \boldsymbol{W}_{1}^{d^{\prime}}\right)^{-1} \int \boldsymbol{W}_{1}^{d} \mathrm{~d} W_{2}}{\left[\int J_{12 c}^{d 2}-\int \boldsymbol{W}_{1}^{d^{\prime}} J_{12 c}^{d}\left(\int \boldsymbol{W}_{1}^{d} \boldsymbol{W}_{1}^{d^{\prime}}\right)^{-1} \int \boldsymbol{W}_{1}^{d} J_{12 c}^{d}\right]^{1 / 2}}
\end{aligned}
$$

For $c=0$, all quantities in Props. 1-3 reduce to the well-known nuisance-parameter free null distributions. More importantly, all limiting functionals are driven by the same Brownian Motions $\boldsymbol{W}$, such that the propositions allow us to consider the joint distribution of the test statistics.

\section{Combination Tests}

Gregory et al. (2004) show that, under $\mathcal{H}_{0}$, many of the above statistics are only weakly correlated, even asymptotically. Further, Pesavento (2004) demonstrates that the tests differ in their power in different parts of the $\left(c-R^{2}\right)$-parameter space. In particular, different tests are most powerful in different parts of the parameter space. As argued in the Introduction, this implies that a more robust, and possibly even more powerful, combination test can in principle be achieved.

Let $t_{i}$ be the test statistic of cointegration test $i=1, \ldots, N$. We define $\xi_{i}:=t_{i}$ if test $i$ rejects for large values and take $-\xi_{i}=t_{i}$ if test $i$ rejects for small values. Define $\Xi_{i}$ as one minus test $i$ 's asymptotic null distribution function, i.e. $\Xi_{i}(x):=\mathrm{P}\left(\xi_{i} \geqslant x\right)$, with $\mathrm{P}$ the probability under $\mathcal{H}_{0}$. The $p$-values of the tests are then given by $p_{i}:=\Xi_{i}\left(\xi_{i}\right)$.

\subsection{A Fisher-type test}

To reach a joint test decision from the different $\xi_{i}$, we require a suitable aggregator. One such aggregator is given Fisher's (1932) famous $\chi^{2}$ test. The following Proposition follows at once from the Continuous Mapping Theorem (CMT). 
Proposition 4. Let $\mathcal{I}$ the index set of the aggregated single $\xi_{i}$. Consider the test statistic

$$
\tilde{\chi}_{\mathcal{I}}^{2}:=-2 \sum_{i \in \mathcal{I}} \ln \left(p_{i}\right)
$$

As $T \rightarrow \infty$, (a) $\tilde{\chi}_{\mathcal{I}}^{2} \rightarrow{ }_{d} \mathcal{F}_{\mathcal{I}}$ under $\mathcal{H}_{0}$, with $\mathcal{F}_{\mathcal{I}}$ some random variable. Further, (b) $\tilde{\chi}_{\mathcal{I}}^{2} \rightarrow \infty$ under $\mathcal{H}_{1}$ if at least one of the underlying tests is consistent, i.e satisfies $p_{i} \rightarrow_{p} 0$ under $\mathcal{H}_{1}$.

Part (a) states that $\tilde{\chi}_{\mathcal{I}}^{2}$ has a well-defined asymptotic null distribution, call it $F_{\mathcal{F}_{\mathcal{I}}}$. The index-set notation $\mathcal{I}$ serves to emphasize that the distribution of the Fisher test depends on which and how many tests are combined. Part $(b)$ establishes the consistency of the $\tilde{\chi}_{\mathcal{I}}^{2}$ tests. Of course we cannot invoke the conventional $\chi^{2}(2|\mathcal{I}|$ ) (with $|\mathcal{I}|$ the cardinality of $\mathcal{I}$ ) null distribution for $\tilde{\chi}_{\mathcal{I}}^{2}$, as independence of the $\xi_{i}, i \in \mathcal{I}$, is necessary for this result. However, focussing on the underlying tests from Propositions 1-3, we can straightforwardly infer and simulate their joint distribution. The aggregator $\tilde{\chi}_{\mathcal{I}}^{2}$ is a continuous function of the $t_{i}$, whose null distribution $F_{\mathcal{F}_{\mathcal{I}}}$ can therefore be derived by simulation of the functional (6). Table 1 reports critical values $F_{\mathcal{F}_{\mathcal{I}}}^{-1}(1-\alpha)$ for combinations of the above-mentioned tests, obtained from 100,000 draws from the distributions $F_{\mathcal{F}_{\mathcal{I}}}$. (From Prop. 4, reject if $\tilde{\chi}_{\mathcal{I}}^{2}>F_{\mathcal{F}_{\mathcal{I}}}^{-1}(1-\alpha)$ ). We approximate the Wiener processes with suitably normalized Gaussian random walks of length $T=1,000$ and tabulate $5 \%$-critical values for several combinations likely to be relevant in practice (see Appendix A for other levels). Moreover, since the distributions of the underlying cointegration tests depend on $K-1$ (reported up to 11) as well as the maintained deterministic specification $(i)$-(iii), that of $\tilde{\chi}_{\mathcal{I}}^{2}$ will not only depend on $\mathcal{I}$ but also on $K-1$ and the maintained case.

We find that, for different combinations, the (5\%-)critical values cluster around 11 for $|\mathcal{I}|=2$, and around 15 for $|\mathcal{I}|=3$. There is little variation across cases. The critical values fall moderately in $K-1$. It is instructive to compare the critical values to those of the $\chi^{2}(2|\mathcal{I}|)$ distribution. The $5 \%$-critical value is 9.487 for $|\mathcal{I}|=2$, and 12.591 for $|\mathcal{I}|=3$. The critical values in Table 1 are uniformly larger. This reflects that the $\xi_{i}$ are generally positively correlated, such that a larger critical value is necessary to construct level- $\alpha$ tests based on (6). Moreover, for each version of $\tilde{\chi}_{\mathcal{I}}^{2}$, the critical values are smaller than $-2 \sum_{i \in \mathcal{I}} \ln (0.05)$ (which e.g. equals 11.983 for $|\mathcal{I}|=2$ ), such that $\tilde{\chi}_{\mathcal{I}}^{2}$ rejects whenever all single tests reject at the $5 \%$-level.

Remark 1. The aggregator (6) is only one of many possible choices. Among others, we aggregate $p$-values using an inverse-normal approach, defined by $1 / \sqrt{|\mathcal{I}|} \sum_{i \in \mathcal{I}} \Phi^{-1}\left(p_{i}\right)$, where $\Phi$ is the distribution function of the standard normal distribution. Its performance was however slightly inferior to that of the $\tilde{\chi}_{\mathcal{I}}^{2}$ tests, to be reported below. Detailed results are available upon request.

Remark 2. We also consider a minimum $p$-value test, given by $\min _{i \in \mathcal{I}} p_{i}$. This test is a direct fix to the 'naive' strategy that rejects whenever one of the single tests rejects. The critical values of the $\min _{i \in \mathcal{I}} p_{i}$ test yield the level $\alpha^{\prime}<\alpha$ at which one needs to test to avoid the oversizedness of the 'naive' approach. Appendix A provides selected correction factors for $\min _{i \in \mathcal{I}} p_{i}$ test. We find $\alpha^{\prime} \gg \alpha /|\mathcal{I}|$. Hence, this test is more powerful than a Bonferroni-type multiple test. 
Table 1: $5 \%$-critical values for the $\tilde{\chi}_{\mathcal{I}}^{2}$ tests

\begin{tabular}{|c|c|c|c|c|c|c|c|c|c|c|c|c|}
\hline \multirow[b]{2}{*}{$K-1$} & \multicolumn{12}{|c|}{ case } \\
\hline & (i) & (ii) & (iii) & (i) & (ii) & (iii) & (i) & (ii) & (iii) & (i) & (ii) & (iii) \\
\hline & \multicolumn{3}{|c|}{$t_{\alpha}^{\mathrm{ADF}}$ and $\lambda_{\max }$} & \multicolumn{3}{|c|}{$\hat{F}$ and $\lambda_{\max }$} & \multicolumn{3}{|c|}{$\hat{F}$ and $t_{\text {ECR }}^{\mathrm{ECR}}$} & \multicolumn{3}{|c|}{$\hat{F}$ and $t_{\nu}^{\mathrm{ADF}}$} \\
\hline 1 & 11.071 & 11.229 & 11.269 & 11.071 & 11.090 & 11.068 & 11.606 & 11.803 & 11.862 & 10.890 & 11.298 & 11.507 \\
\hline 2 & 10.838 & 10.895 & 10.858 & 10.701 & 10.715 & 10.654 & 11.556 & 11.716 & 11.795 & 10.794 & 11.051 & 11.237 \\
\hline 3 & 10.640 & 10.637 & 10.711 & 10.453 & 10.459 & 10.461 & 11.554 & 11.683 & 11.731 & 10.688 & 10.880 & 11.087 \\
\hline 4 & 10.516 & 10.576 & 10.532 & 10.299 & 10.324 & 10.318 & 11.491 & 11.611 & 11.696 & 10.644 & 10.780 & 11.000 \\
\hline 5 & 10.406 & 10.419 & 10.448 & 10.237 & 10.187 & 10.188 & 11.478 & 11.621 & 11.639 & 10.635 & 10.701 & 10.896 \\
\hline 6 & 10.312 & 10.352 & 10.311 & 10.115 & 10.167 & 10.166 & 11.473 & 11.611 & 11.597 & 10.556 & 10.670 & 10.820 \\
\hline 7 & 10.218 & 10.295 & 10.222 & 10.023 & 10.055 & 10.033 & 11.492 & 11.577 & 11.621 & 10.594 & 10.715 & 10.813 \\
\hline 8 & 10.185 & 10.181 & 10.189 & 10.041 & 9.999 & 10.014 & 11.511 & 11.545 & 11.624 & 10.591 & 10.658 & 10.800 \\
\hline 9 & 10.162 & 10.154 & 10.164 & 10.000 & 9.978 & 9.996 & 11.488 & 11.590 & 11.633 & 10.561 & 10.738 & 10.733 \\
\hline 10 & 10.079 & 10.109 & 10.070 & 9.926 & 9.889 & 9.870 & 11.491 & 11.504 & 11.565 & 10.556 & 10.629 & 10.703 \\
\hline \multirow[t]{2}{*}{11} & 10.057 & 10.059 & 10.134 & 9.928 & 9.928 & 9.946 & 11.450 & 11.528 & 11.542 & 10.548 & 10.641 & 10.667 \\
\hline & \multicolumn{3}{|c|}{$\hat{F}, \lambda_{\max }$ and $t_{\gamma}^{\mathrm{ADF}}$} & \multicolumn{3}{|c|}{$\hat{F}, \lambda_{\max }$ and $t_{\gamma}^{\mathrm{ECR}}$} & \multicolumn{3}{|c|}{$\hat{F}, \lambda_{\max }, t_{\gamma}^{\mathrm{ADF}}, t_{\gamma}^{\mathrm{ECR}}$} & & & \\
\hline 1 & 16.037 & 16.363 & 16.582 & 16.287 & 16.572 & 16.633 & 21.352 & 21.931 & 22.215 & & & \\
\hline 2 & 15.526 & 15.732 & 15.856 & 15.827 & 15.927 & 15.965 & 20.776 & 21.106 & 21.342 & & & \\
\hline 3 & 15.186 & 15.294 & 15.471 & 15.440 & 15.512 & 15.620 & 20.237 & 20.486 & 20.788 & & & \\
\hline 4 & 14.934 & 15.025 & 15.173 & 15.184 & 15.291 & 15.407 & 19.951 & 20.143 & 20.440 & & & \\
\hline 5 & 14.720 & 14.825 & 14.990 & 15.045 & 15.092 & 15.260 & 19.747 & 19.888 & 20.170 & & & \\
\hline 6 & 14.578 & 14.685 & 14.833 & 14.924 & 15.056 & 15.155 & 19.564 & 19.761 & 19.934 & & & \\
\hline 7 & 14.472 & 14.612 & 14.632 & 14.852 & 14.964 & 14.946 & 19.471 & 19.688 & 19.722 & & & \\
\hline 8 & 14.460 & 14.427 & 14.595 & 14.823 & 14.825 & 14.941 & 19.471 & 19.447 & 19.678 & & & \\
\hline 9 & 14.332 & 14.405 & 14.496 & 14.766 & 14.801 & 14.872 & 19.365 & 19.492 & 19.582 & & & \\
\hline 10 & 14.321 & 14.322 & 14.301 & 14.717 & 14.733 & 14.775 & 19.268 & 19.365 & 19.398 & & & \\
\hline 11 & 14.230 & 14.300 & 14.357 & 14.696 & 14.773 & 14.824 & 19.151 & 19.345 & 19.404 & & & \\
\hline
\end{tabular}

$5 \%$-critical values for combination tests based on $\tilde{\chi}_{\mathcal{I}}^{2} \cdot t_{\gamma}^{\mathrm{ADF}}$ is from Engle and Granger (1987), $\lambda_{\max }$ from Johansen (1988), $\hat{F}$ from Boswijk (1994) and $t_{\gamma}^{\mathrm{ECR}}$ from Banerjee et al. (1998).

\subsection{Union-of-Rejections tests}

The latter minimum $p$-value test is similar to a recent proposal of Harvey et al. (2009), who develop a 'Union-of-Rejections' $(U R)$ approach to combine standard Dickey-Fuller and GLSdemeaned unit root tests. The $U R$ test also rejects whenever one of the two tests rejects, with however a suitable adjustment of the critical values to ensure a level- $\alpha$ test. This provides a more robust test as the two single tests are relatively more powerful when the initial condition of the time series is large (small). This situation is analogous to the present one, in that $R^{2}$ determines the relative power of the single cointegration tests. We now use and extend the $U R$ approach to the case of cointegration testing considered here.

Denote the single level- $\alpha$ critical value corresponding to the test statistic $\xi_{i}$ as $c v_{i, \alpha}$. The 'naive' $U R$ test statistic for $|\mathcal{I}|=2$ can then be written as

$$
U R^{\text {naive }}\left(\xi_{1}, \xi_{2}\right):=\mathbb{I}\left\{\xi_{1}>c v_{1, \alpha}\right\}+\mathbb{I}\left\{\xi_{1} \leqslant c v_{1, \alpha}\right\} \mathbb{I}\left\{\xi_{2}>c v_{2, \alpha}\right\}
$$

with $\mathbb{I}\{A\}$ the indicator function of event $A$. One would reject $\mathcal{H}_{0}$ if $U R^{\text {naive }}\left(\xi_{1}, \xi_{2}\right)=1$. Of course, the test (7) does not control size. ${ }^{3}$ Harvey et al. (2009) therefore introduce a scaling

\footnotetext{
${ }^{3}$ The null rejection probability of test $i$ is $\operatorname{EI}\left\{\xi_{i}>c v_{i, \alpha}\right\}=\mathrm{P}\left(\xi_{i}>c v_{i, \alpha}\right)=\alpha$. The size of $U R^{\text {naive }}\left(\xi_{1}, \xi_{2}\right)$
} 
constant $\psi$ to modify (7) as follows.

$$
U R_{\psi}\left(\xi_{1}, \xi_{2}\right):=\mathbb{I}\left\{\xi_{1}>\psi c v_{1, \alpha}\right\}+\mathbb{I}\left\{\xi_{1} \leqslant \psi c v_{1, \alpha}\right\} \mathbb{I}\left\{\xi_{2}>\psi c v_{2, \alpha}\right\}
$$

One rejects if $U R_{\psi}\left(\xi_{1}, \xi_{2}\right)=1$, where $\psi$ is unique and to be chosen so that $\mathrm{P}\left(\bigcup_{i=1}^{2} \xi_{i}>\psi c v_{i, \alpha}\right)=\alpha$. However, there is no need to apply the same $\psi$ to both critical values $c v_{i, \alpha}$. In fact, there exists a continuum of tuples of scaling constants so as to obtain a level- $\alpha U R$ test. Define the interval $\mathcal{C}:=\mathbb{R} \cap[1, \infty)$ and let $\widetilde{\boldsymbol{\psi}}:=\left(\widetilde{\psi}_{1}, \widetilde{\psi}_{2}\right) \in \mathcal{C} \times \mathcal{C}=: \mathcal{C}^{2}$. The $U R$ statistic then becomes

$$
U R_{\psi_{\mathcal{I}}}\left(\xi_{1}, \xi_{2}\right):=\mathbb{I}\left\{\xi_{1}>\widetilde{\psi}_{1} c v_{1, \alpha}\right\}+\mathbb{I}\left\{\xi_{1} \leqslant \widetilde{\psi}_{1} c v_{1, \alpha}\right\} \mathbb{I}\left\{\xi_{2}>\widetilde{\psi}_{2} c v_{2, \alpha}\right\}
$$

One rejects if $U R_{\psi_{\mathcal{I}}}\left(\xi_{1}, \xi_{2}\right)=1$. The admissible tuples $\widetilde{\boldsymbol{\psi}}$, denoted $\boldsymbol{\psi}$, are implicitly defined by

$$
\mathrm{P}\left(\bigcup_{i=1}^{2} \xi_{i}>\psi_{i} c v_{i, \alpha}\right)=\alpha
$$

The $\boldsymbol{\psi}$ are again identified in the sense that, for each $\psi_{1} \in \mathcal{C}$, there exists exactly one $\psi_{2} \in \mathcal{C}$ such that (10) holds. The solution $\psi=\psi_{1}=\psi_{2}$ considered by Harvey et al. (2009) is thus a special case of (10). In contrast, condition (10) defines an entire family of tests.

Remark 3. Searching over $\mathcal{C}^{2}$ is without loss of generality. Suppose $\widetilde{\psi}_{1}<1$. We then have $\mathrm{P}\left(\xi_{1}>\widetilde{\psi}_{1} c v_{1, \alpha}\right)=: \widetilde{\alpha}_{1}>\alpha$. Also write $\mathrm{P}\left(\xi_{2}>\widetilde{\psi}_{2} c v_{2, \alpha}\right)=: \widetilde{\alpha}_{2}$. It obtains that (cf. fn. 3) $\mathrm{P}\left(\bigcup_{i=1}^{2} \xi_{i}>\widetilde{\psi}_{i} c v_{i, \alpha}\right)=\widetilde{\alpha}_{1}+\widetilde{\alpha}_{2}-\mathrm{P}\left(\bigcap_{i=1}^{2} \xi_{i}>\widetilde{\psi}_{i} c v_{i, \alpha}\right) \geqslant \widetilde{\alpha}_{1}>\alpha$, because $\mathrm{P}\left(\bigcap_{i=1}^{2} \xi_{i}>\widetilde{\psi}_{i} c v_{i, \alpha}\right) \leqslant$ $\widetilde{\alpha}_{2}$. Hence, one cannot make one test more liberal and still achieve a level- $\alpha U R_{\psi_{\mathcal{I}}}$ test.

The availability of an entire family of level- $\alpha$ tests, indexed by $\boldsymbol{\psi}$, raises the practical question of which $\boldsymbol{\psi}$ to select. There is no unique uniformly most powerful choice. We propose to select $\boldsymbol{\psi}$ such that, subject to (10),

$$
\psi_{1}=\underset{\widetilde{\psi}_{1} \in \mathcal{C}}{\arg \min }\left\{\frac{\mathrm{P}\left(\xi_{1}>\widetilde{\psi}_{1} c v_{1, \alpha} \cap \xi_{2}>\psi_{2} c v_{i, \alpha}\right)}{\min \left\{\mathrm{P}\left(\xi_{1}>\widetilde{\psi}_{1} c v_{1, \alpha}\right), \mathrm{P}\left(\xi_{2}>\psi_{2} c v_{2, \alpha}\right)\right\}}\right\}
$$

It is sufficient to minimize over $\psi_{1}$ only, since the corresponding $\psi_{2}$ is uniquely determined by (10). ${ }^{4}$ We refer to this member of the family of tests as the 'asymmetric' $U R$ test. The tuples $\boldsymbol{\psi}$ for the test pairs $t_{\gamma}^{\mathrm{ADF}}$ and $\lambda_{\max }, \hat{F}$ and $\lambda_{\max }$ as well as $\hat{F}$ and $t_{\gamma}^{\mathrm{ECR}}$ for $K-1$ up to 11 are reported in Table 2. This decision rule can be expected to yield powerful $U R_{\psi_{\mathcal{I}}}$ tests as (11) minimizes the number of instances where both tests reject under $\mathcal{H}_{0}$, while still generating a level- $\alpha$ test. That is, the tests are made as 'uncorrelated' as possible, without violating constraint (10). Now, since the behavior of the tests under local alternatives will change continuously from that under therefore equals $\mathrm{P}\left(\bigcup_{i=1}^{2} \xi_{i}>c v_{i, \alpha}\right)=\mathrm{P}\left(\xi_{1}>c v_{1, \alpha}\right)+\mathrm{P}\left(\xi_{2}>c v_{2, \alpha}\right)-\mathrm{P}\left(\bigcap_{i=1}^{2} \xi_{i}>c v_{i, \alpha}\right)=2 \alpha-\mathrm{P}\left(\bigcap_{i=1}^{2} \xi_{i}>\right.$ $\left.c v_{i, \alpha}\right) \geqslant \alpha$, since $\mathrm{P}\left(\bigcap_{i=1}^{2} \xi_{i}>c v_{i, \alpha}\right) \leqslant \mathrm{P}\left(\xi_{i}>c v_{i, \alpha}\right)=\alpha$.

${ }^{4}$ We add an $\epsilon$ to the numerator of (11) to penalize borderline cases in which, due to simulation imprecision of the Wiener integrals, the numerator would otherwise be zero and the denominator very small, but positive. 
Table 2: Correction Factors for the $U R_{\psi_{\mathcal{I}}}$ test

\begin{tabular}{|c|c|c|c|c|c|c|c|c|c|c|}
\hline \multirow[b]{2}{*}{$K-1$} & \multirow[b]{2}{*}{ case } & \multicolumn{3}{|c|}{$t_{\gamma}^{\mathrm{ADF}}$ and $\lambda_{\max }$} & \multicolumn{3}{|c|}{$\hat{F}$ and $\lambda_{\max }$} & \multicolumn{3}{|c|}{$\hat{F}$ and $t_{\gamma}^{\mathrm{ECR}}$} \\
\hline & & (i) & (ii) & (iii) & (i) & (ii) & (iii) & (i) & (ii) & (iii) \\
\hline & & \multicolumn{3}{|c|}{$t_{\gamma}^{\mathrm{ADF}}$} & \multicolumn{3}{|c|}{$\hat{F}$} & \multicolumn{3}{|c|}{$\hat{F}$} \\
\hline 1 & & 1.065 & 1.050 & 1.043 & 1.128 & 1.104 & 1.093 & 1.077 & 1.042 & 1.032 \\
\hline 2 & & 1.058 & 1.052 & 1.044 & 1.131 & 1.110 & 1.095 & 1.075 & 1.052 & 1.038 \\
\hline 3 & & 1.055 & 1.049 & 1.046 & 1.122 & 1.104 & 1.096 & 1.070 & 1.053 & 1.038 \\
\hline 4 & & 1.051 & 1.045 & 1.042 & 1.107 & 1.099 & 1.090 & 1.057 & 1.053 & 1.043 \\
\hline 5 & & 1.048 & 1.045 & 1.041 & 1.103 & 1.094 & 1.088 & 1.058 & 1.049 & 1.043 \\
\hline 6 & & 1.046 & 1.044 & 1.040 & 1.096 & 1.091 & 1.085 & 1.060 & 1.051 & 1.044 \\
\hline 7 & & 1.045 & 1.042 & 1.035 & 1.092 & 1.082 & 1.082 & 1.056 & 1.055 & 1.045 \\
\hline 8 & & 1.042 & 1.041 & 1.039 & 1.089 & 1.080 & 1.081 & 1.050 & 1.044 & 1.044 \\
\hline 9 & & 1.040 & 1.038 & 1.039 & 1.085 & 1.081 & 1.078 & 1.049 & 1.047 & 1.044 \\
\hline 10 & & 1.039 & 1.035 & 1.037 & 1.079 & 1.008 & 1.075 & 1.046 & 1.041 & 1.043 \\
\hline \multirow{2}{*}{\multicolumn{2}{|c|}{11}} & 1.038 & 1.037 & 1.035 & 1.072 & 1.076 & 1.071 & 1.047 & 1.045 & 1.041 \\
\hline & & \multicolumn{3}{|c|}{$\lambda_{\max }$} & \multicolumn{3}{|c|}{$\lambda_{\max }$} & \multicolumn{3}{|c|}{$t_{\gamma}^{\mathrm{ECR}}$} \\
\hline 1 & & 1.100 & 1.077 & 1.065 & 1.101 & 1.083 & 1.070 & 1.049 & 1.022 & 1.018 \\
\hline 2 & & 1.080 & 1.076 & 1.068 & 1.084 & 1.082 & 1.075 & 1.046 & 1.028 & 1.023 \\
\hline 3 & & 1.074 & 1.063 & 1.064 & 1.075 & 1.067 & 1.068 & 1.046 & 1.033 & 1.023 \\
\hline 4 & & 1.066 & 1.059 & 1.056 & 1.071 & 1.063 & 1.061 & 1.042 & 1.033 & 1.028 \\
\hline 5 & & 1.061 & 1.055 & 1.053 & 1.063 & 1.058 & 1.055 & 1.040 & 1.032 & 1.029 \\
\hline 6 & & 1.052 & 1.051 & 1.052 & 1.056 & 1.052 & 1.054 & 1.041 & 1.034 & 1.028 \\
\hline 7 & & 1.049 & 1.047 & 1.054 & 1.050 & 1.053 & 1.049 & 1.039 & 1.035 & 1.029 \\
\hline 8 & & 1.045 & 1.045 & 1.043 & 1.047 & 1.048 & 1.045 & 1.036 & 1.032 & 1.028 \\
\hline 9 & & 1.045 & 1.042 & 1.043 & 1.044 & 1.042 & 1.046 & 1.034 & 1.032 & 1.028 \\
\hline 10 & & 1.043 & 1.043 & 1.038 & 1.044 & 1.161 & 1.039 & 1.034 & 1.031 & 1.030 \\
\hline 11 & & 1.040 & 1.039 & 1.037 & 1.043 & 1.039 & 1.039 & 1.035 & 1.032 & 1.028 \\
\hline
\end{tabular}

See notes to Table 1.

$\mathcal{H}_{0}$, making the tests 'uncorrelated' will produce a high number of rejections under $\mathcal{H}_{1} \cdot{ }^{5}$

Remark 4. It turns out that the selection rule (11) satisfies

$$
\mathrm{P}\left(\xi_{1}>\psi_{1} c v_{1, \alpha}\right)=\mathrm{P}\left(\xi_{2}>\psi_{2} c v_{2, \alpha}\right)
$$

for all combinations considered in Table $2{ }^{6}$ Under this condition, the $U R_{\psi_{\mathcal{I}}}$ test is equivalent to the min-test described in Remark 2. To show this, we first show that the min-test belongs to the family of $U R_{\psi_{\mathcal{I}}}$ tests. Let $F_{\min }$ be the null distribution function of $\min \left(p_{1}, p_{2}\right)$. The min-test rejects if $\min \left(p_{1}, p_{2}\right)<F_{\min }^{-1}(\alpha)$, thus if $p_{1}<F_{\min }^{-1}(\alpha) \vee p_{2}<F_{\min }^{-1}(\alpha)$. Equivalently, the test rejects if $\Xi_{1}^{-1}\left(p_{1}\right)>\Xi_{1}^{-1}\left(F_{\min }^{-1}(\alpha)\right) \vee \Xi_{2}^{-1}\left(p_{2}\right)>\Xi_{2}^{-1}\left(F_{\min }^{-1}(\alpha)\right)$ (recall the $\Xi_{i}$ are defined to be

\footnotetext{
${ }^{5}$ Unreported experiments with other tuples confirm this conjecture.

${ }^{6}$ To see why, write the numerator of $(11)$ as $\mathrm{P}\left(\xi_{1}>\psi_{1} c v_{1, \alpha}\right)+\mathrm{P}\left(\xi_{2}>\psi_{2} c v_{2, \alpha}\right)-\mathrm{P}\left(\bigcup_{i=1}^{2} \xi_{i}>\psi_{i} c v_{i, \alpha}\right)$. W.l.o.g. take the denominator to equal $\mathrm{P}\left(\xi_{1}>\psi_{1} c v_{1, \alpha}\right)$. Using that $\mathrm{P}\left(\bigcup_{i=1}^{2} \xi_{i}>\psi_{i} c v_{i, \alpha}\right)=\alpha$ for solutions to (10), (11) equals $\min _{\psi_{1}}\left[1+\left\{\mathrm{P}\left(\xi_{2}>\psi_{2} c v_{2, \alpha}\right)-\alpha\right\} / \mathrm{P}\left(\xi_{1}>\psi_{1} c v_{1, \alpha}\right)\right]$. Taking the derivative w.r.t. $\mathrm{P}\left(\xi_{1}>\psi_{1} c v_{1, \alpha}\right)$ yields$$
\frac{\partial \mathrm{P}\left(\xi_{2}>\psi_{2} c v_{2, \alpha}\right) / \partial \mathrm{P}\left(\xi_{1}>\psi_{1} c v_{1, \alpha}\right) \mathrm{P}\left(\xi_{1}>\psi_{1} c v_{1, \alpha}\right)-\left[\mathrm{P}\left(\xi_{2}>\psi_{2} c v_{2, \alpha}\right)-\alpha\right]}{\mathrm{P}\left(\xi_{1}>\psi_{1} c v_{1, \alpha}\right)^{2}},
$$

which has an interior minimum (i.e. $\mathrm{P}\left(\xi_{1}>\psi_{1} c v_{1, \alpha}\right)<\mathrm{P}\left(\xi_{2}>\psi_{2} c v_{2, \alpha}\right)$ strictly) if $(*)$ equals zero. That is, the 'indifference curves' generated by the solutions $\boldsymbol{\psi}$ to (10) are sufficiently steep to produce the 'corner solution' (12).
} 
decreasing functions). Since $p_{i}=\Xi_{i}\left(\xi_{i}\right)$, this test thus rejects if and only if

$$
\xi_{1}>\Xi_{1}^{-1}\left(F_{\min }^{-1}(\alpha)\right) \quad \vee \quad \xi_{2}>\Xi_{2}^{-1}\left(F_{\min }^{-1}(\alpha)\right)
$$

or equivalently if

$$
\xi_{1}>\psi_{1} c v_{1, \alpha} \quad \vee \quad \xi_{2}>\psi_{2} c v_{2, \alpha} .
$$

where $\psi_{i}:=\Xi_{i}^{-1}\left(F_{\min }^{-1}(\alpha)\right) / c v_{i, \alpha}$. We know that, under $\mathcal{H}_{0}, \mathrm{P}\left(\xi_{1}>\Xi_{1}^{-1}\left(F_{\min }^{-1}(\alpha)\right) \vee \xi_{2}>\right.$ $\left.\Xi_{2}^{-1}\left(F_{\min }^{-1}(\alpha)\right)\right)=\alpha$, so that the min-test is a $U R_{\psi_{\mathcal{I}}}$ test. It remains to establish that the min-test is the only $U R_{\psi_{\mathcal{I}}}$ test that satisfies (12). By construction,

$$
\mathrm{P}\left(\xi_{i}>\psi_{i} c v_{i, \alpha}\right)=\mathrm{P}\left(\xi_{i}>\Xi_{i}^{-1}\left(F_{\min }^{-1}(\alpha)\right)\right)=F_{\min }^{-1}(\alpha) \quad i=1,2 .
$$

Uniqueness follows from monotonicity of the $\Xi_{i}$.

Remark 5. One can furthermore relax another of Harvey et al.'s restrictions, viz. that of combining $|\mathcal{I}|=2$ tests. An $|\mathcal{I}|$-dimensional $U R$ test is then, analogously to (9), defined by

$$
\mathrm{P}\left(\bigcup_{i=1}^{|\mathcal{I}|} \xi_{i}>\psi_{i} c v_{i, \alpha}\right)=\alpha .
$$

Of course, the detection of the solution $\psi \in \mathcal{C}^{|\mathcal{I}|}$ then generally becomes numerically more challenging. For the symmetrical solution $\psi=\psi_{1}=\psi_{2}=\psi_{3}$ of $|\mathcal{I}|=3$, where the tests considered are $\hat{F}, \lambda_{\max }$ and $t_{\gamma}^{\mathrm{ADF}}$, we find a similar performance to the tests with $|\mathcal{I}|=2$ discussed above, and therefore do not report detailed results for brevity.

\section{Large Sample Results}

We now report the large-sample power of the tests discussed in the previous sections. The power functions are computed as the probability that the statistics $\xi_{i}$ and $\tilde{\chi}_{\mathcal{I}}^{2}$ exceed their level- $\alpha$ critical value, and the probability that the $U R_{\psi_{\mathcal{I}}}\left(\xi_{1}, \xi_{2}\right)$ test (9) rejects. Given Propositions 1-3 and the results from Section 3, the asymptotic local power can be approximated by simulating the distributions presented above. We draw 15,000 replications of the functionals, for $T=1,000$. We put $c \in\{-1,-2,-3, \ldots,-30\}$ for the local-to-unity parameter and generate $R^{2}$ from $\{0,0.05,0.1, \ldots, 0.95\}$. The number of regressors $K-1$ ranges from 1 to 5 .

Table 3 reports the local asymptotic power of several combination tests as well as the corresponding single tests for case (ii) (see Appendix B for the other cases). Figures 1-2 plot the tests' power against $R^{2}$, for $c=-10$ and $c=-15$. We report results for $K-1=1$; additional results are available upon request. We replicate Pesavento's finding that $t_{\gamma}^{\mathrm{ECR}}$ is the best single test for small $R^{2}$. The power of all tests, with the exception of $t_{\gamma}^{\mathrm{ADF}}$, increases quite quickly in $R^{2}$. The system-based $\lambda_{\max }$ test benefits most from an increase in $R^{2}$, fully exploiting the additional information contained in the equations for the $\boldsymbol{x}_{t}$. The formal similarity of $\hat{F}$ and $t_{\gamma}^{\mathrm{ECR}}$ translates 
into similar local asymptotic power. The combination tests perform very well, in that they track the better of the underlying tests very closely. Their power curves sometimes even lie above that of the underlying tests. This effect is best seen in the lower panels, where the performance of the underlying tests $t_{\gamma}^{\mathrm{ADF}}$ and $\lambda_{\max }$ differs strongly. The upper panels show that, unsurprisingly, the power of the combination tests differs relatively less from that of either of the underlying tests if these perform similarly. Yet, $U R_{\psi_{\mathcal{I}}}\left(\hat{F}, t_{\gamma}^{\mathrm{ECR}}\right)$ and $\tilde{\chi}_{\mathcal{I}}^{2}\left(\hat{F}, t_{\gamma}^{\mathrm{ECR}}\right)$ are again closer to the better underlying test (typically $\hat{F}$ ) whenever there are discernible differences.

Figures 3-5 plot the tests' power against $-c$, holding $R^{2}$ fixed at $0,0.25$ and 0.7 . All tests become more powerful as the distance $c$ to $\mathcal{H}_{0}$ increases, although the speed differs substantially. For large $R^{2}$ and $c=-15$, the power of $\lambda_{\max }, \tilde{\chi}_{\mathcal{I}}^{2}\left(t_{\gamma}^{\mathrm{ADF}}, \lambda_{\max }\right)$ and $U R_{\psi_{\mathcal{I}}}\left(t_{\gamma}^{\mathrm{ADF}}, \lambda_{\max }\right)$ is more than three times larger than that of $t_{\gamma}^{\mathrm{ADF}}$. It is again readily apparent that the combination tests are always close to the better of the two combined single tests. Of course, when the difference between the single tests is large, as in the lower panel of Figure 5, the power distance of the combination tests to the best single tests is somewhat larger. However, the combination tests' power is much closer to that of the better single test. Thus, the combination tests effectively offer a cheap insurance against selecting an inferior test, in that one never sacrifices much power, and potentially gains a lot. Moreover, for $R^{2}=0.25$, both the $\tilde{\chi}_{\mathcal{I}}^{2}\left(t_{\gamma}^{\mathrm{ADF}}, \lambda_{\max }\right)$ test and the corresponding $U R_{\psi_{\mathcal{I}}}$ test even outperform both constituent single tests. Note from Figures 1-2 (the effect is more apparent in Fig. 2) that the power curves of the constituent tests $t_{\gamma}^{\mathrm{ADF}}$ and $\lambda_{\max }$ intersect at $R^{2} \approx 0.25$. Thus, combination tests appear to outperform the constituent tests when the latter are equally powerful. This effect becomes more pronounced with increasing $K-1$, cf. Figures 6 and 4 .

Comparing the performance of $\tilde{\chi}_{\mathcal{I}}^{2}$ and $U R_{\psi_{\mathcal{I}}}$, we find that the former are somewhat more powerful when both constituent tests have relatively high power. The $U R_{\psi_{\mathcal{I}}}$ tests outperform the $\tilde{\chi}_{\mathcal{I}}^{2}$ tests when there is a large difference in power between the single tests, in particular if the weaker one has low absolute power. This is intuitive as $U R_{\psi_{\mathcal{I}}}$ looks for (at least) one single test indicating that $\mathcal{H}_{1}$ holds, effectively ignoring the less powerful test once the more powerful underlying one rejects. On the other hand, $\tilde{\chi}_{\mathcal{I}}^{2}$ combines evidence from both tests, such that a test with low power can tilt the decision of $\tilde{\chi}_{\mathcal{I}}^{2}$ towards a non-rejection of $\mathcal{H}_{0}$. If both tests are at least moderately powerful, $\tilde{\chi}_{\mathcal{I}}^{2}$ will combine that evidence to produce a rejection of $\mathcal{H}_{0}$.

Remark 6. As discussed above, some single tests are most powerful when $R^{2}$ is low, and others when $R^{2}$ is large. This might, alternatively to the approach discussed here, suggest a pretest strategy where one first estimates $R^{2}$ and then selects the most powerful cointegration test given the estimate $\hat{R}^{2}$. However, as pointed out by Pesavento (2007), because (unlike in Elliott et al., 2005) $\boldsymbol{\theta}$ is assumed unknown and several quantities are not consistently estimable in the present local-to-unity framework, it is not clear whether such an estimator $\hat{R}^{2}$ is feasible at all. Moreover, the above results show that the combination tests are never much less, and sometimes even more, powerful than the best single test. They are generally a lot more powerful than the worst test. Thus, even if an estimator $\hat{R}^{2}$ was available, it would not, certainly not for $T$ finite, estimate $R^{2}$ without error, such that a pretest would sometimes select the less powerful 
Table 3: Local Asymptotic Power

\begin{tabular}{|c|c|c|c|c|c|}
\hline$-c$ & 0 & 5 & 10 & 15 & 20 \\
\hline \multicolumn{6}{|l|}{$R^{2}=0$} \\
\hline$\tilde{\chi}_{\mathcal{I}}^{2}\left(\hat{F}, t_{\gamma}^{\mathrm{ECR}}\right)$ & 0.050 & 0.106 & 0.240 & 0.455 & 0.706 \\
\hline$\tilde{\chi}_{\mathcal{I}}^{2}\left(t_{\gamma}^{\mathrm{ADF}}, \lambda_{\max }\right)$ & 0.050 & 0.090 & 0.189 & 0.365 & 0.605 \\
\hline$\tilde{\chi}_{\mathcal{I}}^{2}\left(\hat{F}, t_{\gamma}^{\mathrm{ECR}}, t_{\gamma}^{\mathrm{ADF}}, \lambda_{\max }\right)$ & 0.050 & 0.107 & 0.239 & 0.450 & 0.699 \\
\hline$U R_{\psi_{\mathcal{I}}}\left(\hat{F}, t_{\gamma}^{\mathrm{ECR}}\right)$ & 0.050 & 0.102 & 0.229 & 0.440 & 0.690 \\
\hline$U R_{\psi_{\mathcal{I}}}\left(t_{\gamma}^{\mathrm{ADF}}, \lambda_{\max }\right)$ & 0.050 & 0.080 & 0.171 & 0.334 & 0.571 \\
\hline$\hat{F}$ & 0.050 & 0.096 & 0.212 & 0.408 & 0.657 \\
\hline$t_{\gamma}^{\mathrm{ECR}}$ & 0.050 & 0.112 & 0.255 & 0.482 & 0.731 \\
\hline$\lambda_{\max }$ & 0.050 & 0.068 & 0.124 & 0.239 & 0.427 \\
\hline$t_{\gamma}^{\mathrm{ADF}}$ & 0.050 & 0.098 & 0.221 & 0.422 & 0.674 \\
\hline \multicolumn{6}{|l|}{$R^{2}=0.25$} \\
\hline$\tilde{\chi}_{\mathcal{I}}^{2}\left(\hat{F}, t_{\gamma}^{\mathrm{ECR}}\right)$ & 0.051 & 0.116 & 0.320 & 0.623 & 0.858 \\
\hline$\tilde{\chi}_{\mathcal{I}}^{2}\left(t_{\gamma}^{\mathrm{ADF}}, \lambda_{\max }\right)$ & 0.051 & 0.083 & 0.198 & 0.434 & 0.712 \\
\hline$\tilde{\chi}_{\mathcal{I}}^{2}\left(\hat{F}, t_{\gamma}^{\mathrm{ECR}}, t_{\gamma}^{\mathrm{ADF}}, \lambda_{\max }\right)$ & 0.053 & 0.108 & 0.285 & 0.580 & 0.836 \\
\hline$U R_{\psi_{\mathcal{I}}}\left(\hat{F}, t_{\gamma}^{\mathrm{ECR}}\right)$ & 0.051 & 0.114 & 0.310 & 0.609 & 0.846 \\
\hline$U R_{\psi_{\mathcal{I}}}\left(t_{\gamma}^{\mathrm{ADF}}, \lambda_{\max }\right)$ & 0.051 & 0.081 & 0.186 & 0.399 & 0.661 \\
\hline$\hat{F}$ & 0.053 & 0.117 & 0.317 & 0.614 & 0.845 \\
\hline$t_{\gamma}^{\mathrm{ECR}}$ & 0.050 & 0.114 & 0.308 & 0.613 & 0.853 \\
\hline$\lambda_{\max }$ & 0.051 & 0.078 & 0.185 & 0.402 & 0.662 \\
\hline$t_{\gamma}^{\mathrm{ADF}}$ & 0.051 & 0.081 & 0.177 & 0.360 & 0.603 \\
\hline \multicolumn{6}{|l|}{$R^{2}=0.5$} \\
\hline$\tilde{\chi}_{\mathcal{I}}^{2}\left(\hat{F}, t_{\gamma}^{\mathrm{ECR}}\right)$ & 0.052 & 0.145 & 0.506 & 0.832 & 0.966 \\
\hline$\tilde{\chi}_{\mathcal{I}}^{2}\left(t_{\gamma}^{\mathrm{ADF}}, \lambda_{\max }\right)$ & 0.051 & 0.080 & 0.268 & 0.618 & 0.897 \\
\hline$\tilde{\chi}_{\mathcal{I}}^{2}\left(\hat{F}, t_{\gamma}^{\mathrm{ECR}}, t_{\gamma}^{\mathrm{ADF}}, \lambda_{\max }\right)$ & 0.052 & 0.120 & 0.434 & 0.792 & 0.965 \\
\hline$U R_{\psi_{\mathcal{I}}}\left(\hat{F}, t_{\gamma}^{\mathrm{ECR}}\right)$ & 0.053 & 0.158 & 0.517 & 0.831 & 0.964 \\
\hline$U R_{\psi_{\mathcal{I}}}\left(t_{\gamma}^{\mathrm{ADF}}, \lambda_{\max }\right)$ & 0.051 & 0.092 & 0.307 & 0.639 & 0.892 \\
\hline$\hat{F}$ & 0.055 & 0.171 & 0.539 & 0.842 & 0.966 \\
\hline$t_{\gamma}^{\mathrm{ECR}}$ & 0.050 & 0.124 & 0.444 & 0.792 & 0.957 \\
\hline$\lambda_{\max }$ & 0.052 & 0.109 & 0.360 & 0.699 & 0.922 \\
\hline$t_{\gamma}^{\mathrm{ADF}}$ & 0.051 & 0.061 & 0.135 & 0.292 & 0.527 \\
\hline \multicolumn{6}{|l|}{$R^{2}=0.75$} \\
\hline$\tilde{\chi}_{\mathcal{I}}^{2}\left(\hat{F}, t_{\gamma}^{\mathrm{ECR}}\right)$ & 0.052 & 0.300 & 0.834 & 0.983 & 0.999 \\
\hline$\tilde{\chi}_{\mathcal{I}}^{2}\left(t_{\gamma}^{\mathrm{ADF}}, \lambda_{\max }\right)$ & 0.054 & 0.128 & 0.613 & 0.954 & 0.999 \\
\hline$\tilde{\chi}_{\mathcal{I}}^{2}\left(\hat{F}, t_{\gamma}^{\mathrm{ECR}}, t_{\gamma}^{\mathrm{ADF}}, \lambda_{\max }\right)$ & 0.056 & 0.238 & 0.795 & 0.985 & 1.000 \\
\hline$U R_{\psi_{\mathcal{I}}}\left(\hat{F}, t_{\gamma}^{\mathrm{ECR}}\right)$ & 0.054 & 0.365 & 0.859 & 0.985 & 0.999 \\
\hline$U R_{\psi_{\mathcal{I}}}\left(t_{\gamma}^{\mathrm{ADF}}, \lambda_{\max }\right)$ & 0.052 & 0.212 & 0.738 & 0.973 & 1.000 \\
\hline$\hat{F}$ & 0.056 & 0.391 & 0.872 & 0.987 & 0.999 \\
\hline$t_{\gamma}^{\mathrm{ECR}}$ & 0.050 & 0.197 & 0.718 & 0.957 & 0.997 \\
\hline$\lambda_{\max }$ & 0.053 & 0.267 & 0.798 & 0.984 & 1.000 \\
\hline$t_{\gamma}^{\mathrm{ADF}}$ & 0.053 & 0.039 & 0.083 & 0.210 & 0.433 \\
\hline
\end{tabular}

Case $(i i) . \quad \tilde{\chi}_{\mathcal{I}}^{2}\left(\hat{F}, t_{\gamma}^{\mathrm{ECR}}\right)$ is our Fisher test (6) based on Boswijk's and Banerjee et al.'s tests, and $U R_{\psi_{\mathcal{I}}}\left(\hat{F}, t_{\gamma}^{\mathrm{ECR}}\right)$ is the corresponding Union-of-Rejections test (9). The other combination tests are defined analogously. See also notes to Table 1. 
Figure 1: Local asymptotic power as a function of $R^{2}, c=-10$
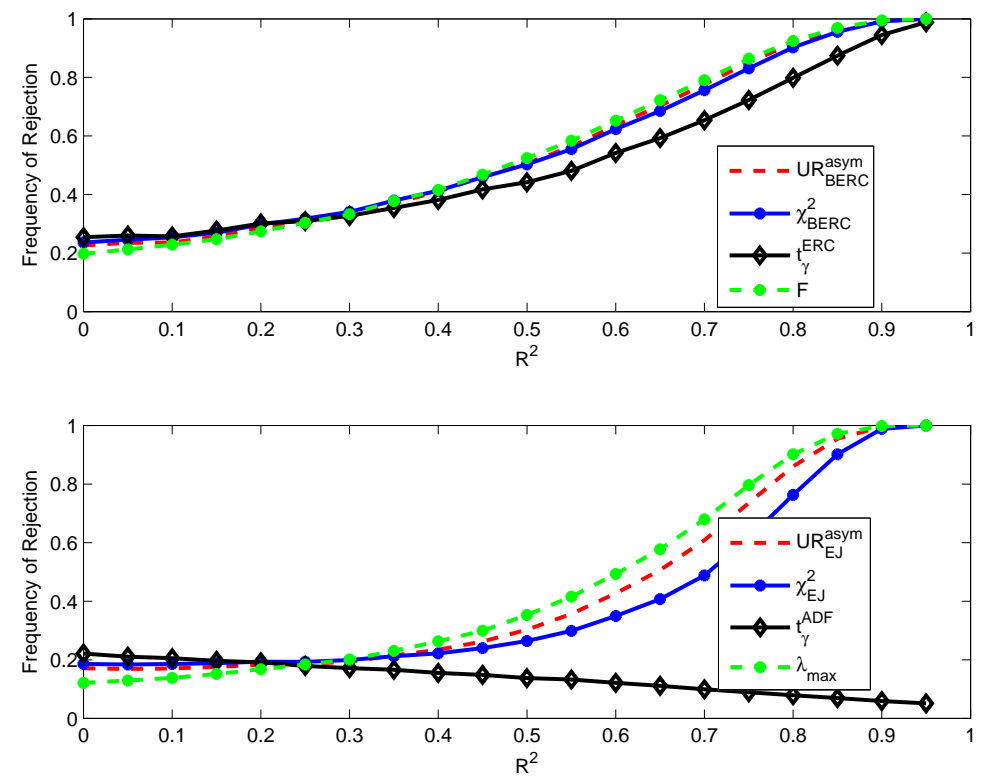

Figure 2: Local asymptotic power as a function of $R^{2}, c=-15$
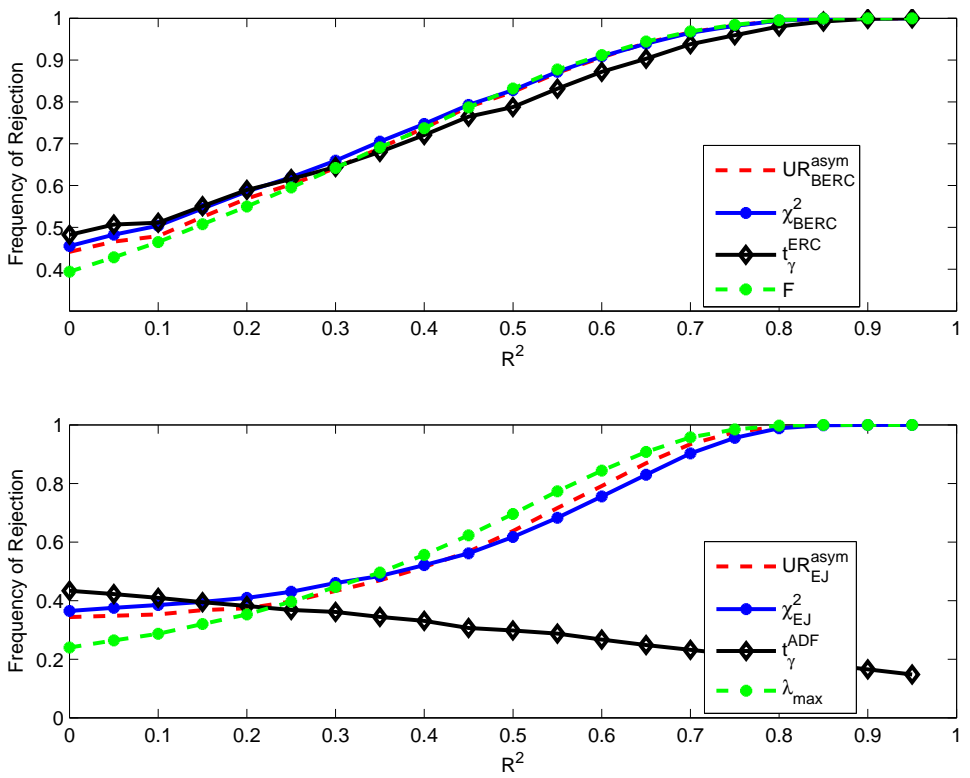

Results are for the demeaned case (ii). $\chi_{\text {BERC }}^{2}$ is our Fisher test (6) based on Boswijk's and Banerjee et al.'s tests. $\chi_{\mathrm{EJ}}^{2}$ is based on Engle and Granger's and Johansen's tests. $U R_{\mathrm{BERC}}^{\mathrm{asym}}$ and $U R_{\mathrm{EJ}}^{\mathrm{asym}}$ are the corresponding asymmetric $U R_{\psi_{\mathcal{I}}}$ tests (9). The single tests' power curves are for comparison. 
Figure 3: Local asymptotic power as a function of $-c, R^{2}=0$
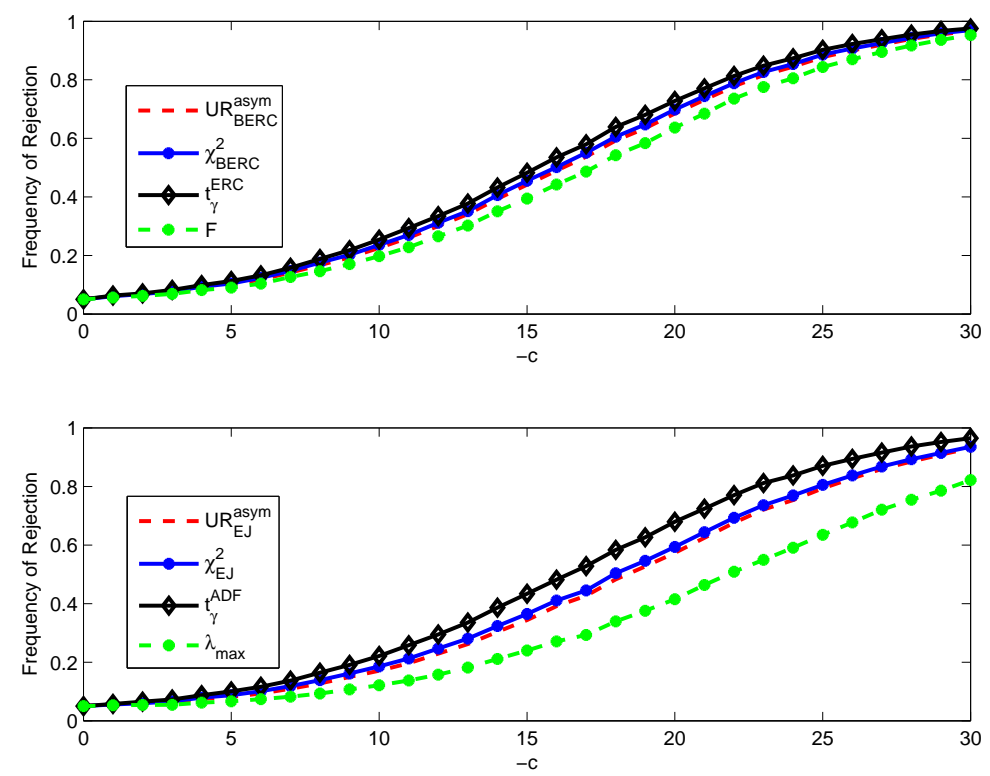

Figure 4: Local asymptotic power as a function of $-c, R^{2}=0.25$
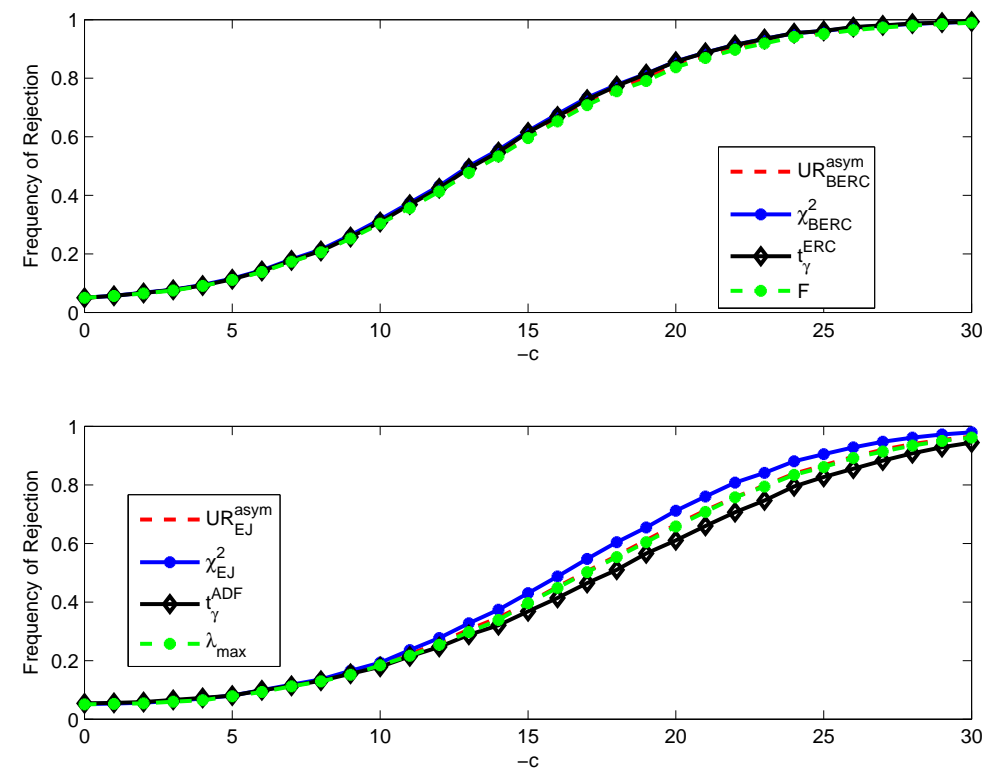

See notes to Figure 1. 
Figure 5: Local asymptotic power as a function of $-c, R^{2}=0.7$
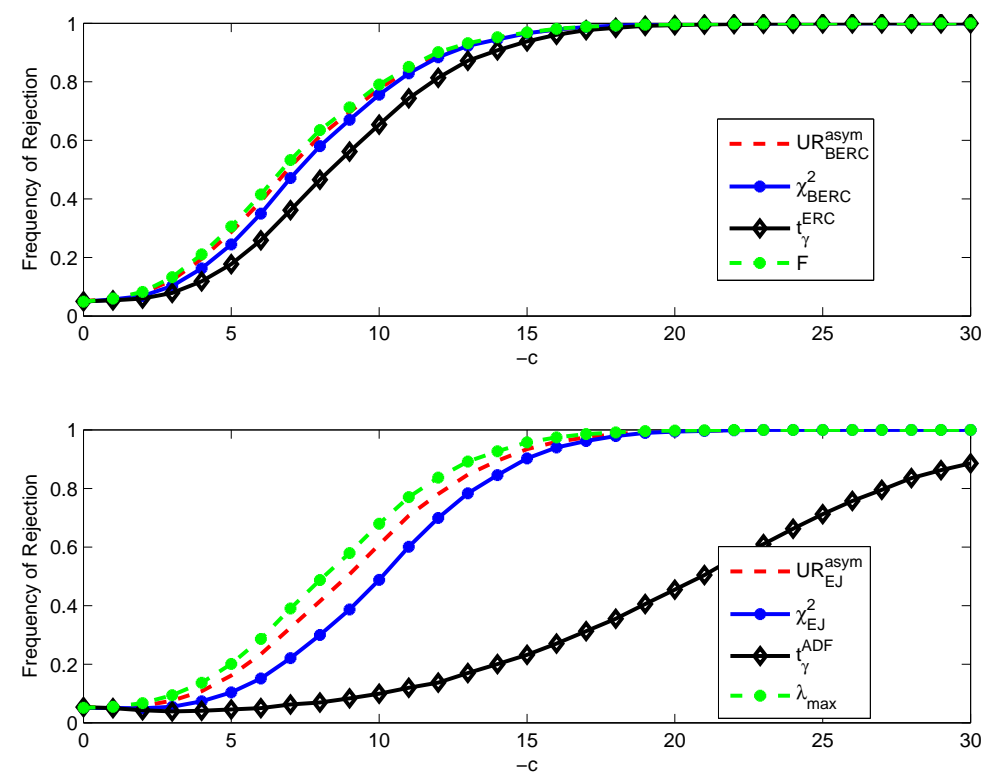

Figure 6: Local asymptotic power as a function of $c, R^{2}=0.35, K-1=3$
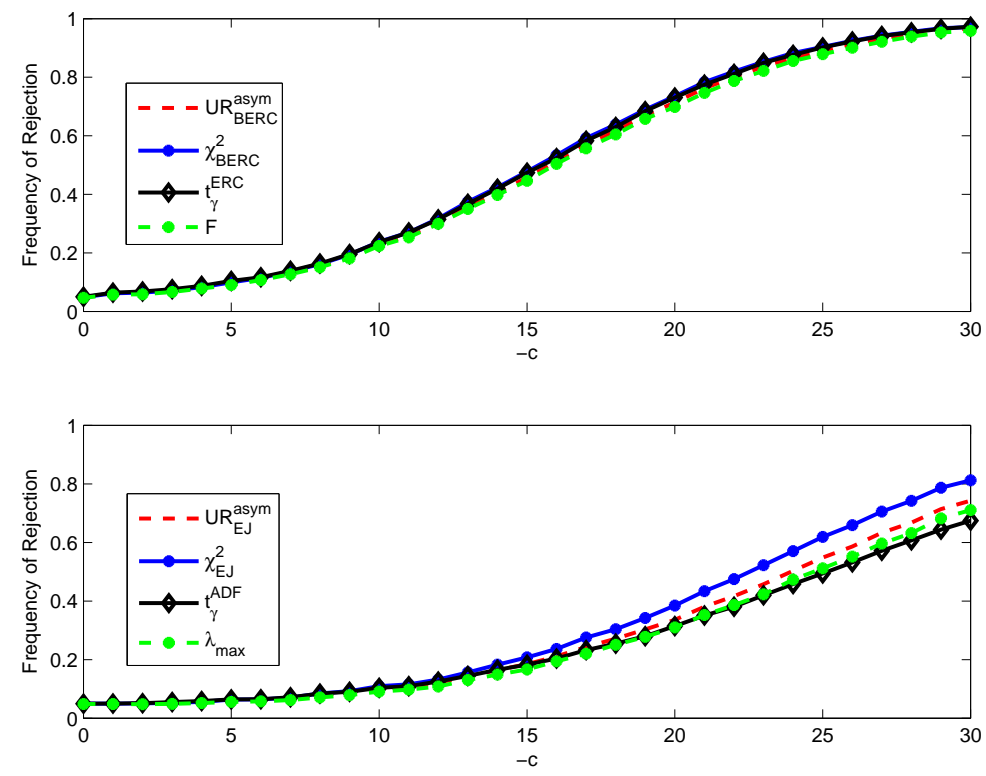

See notes to Figure 1. 
Figure 7: Cutoff probability $q$

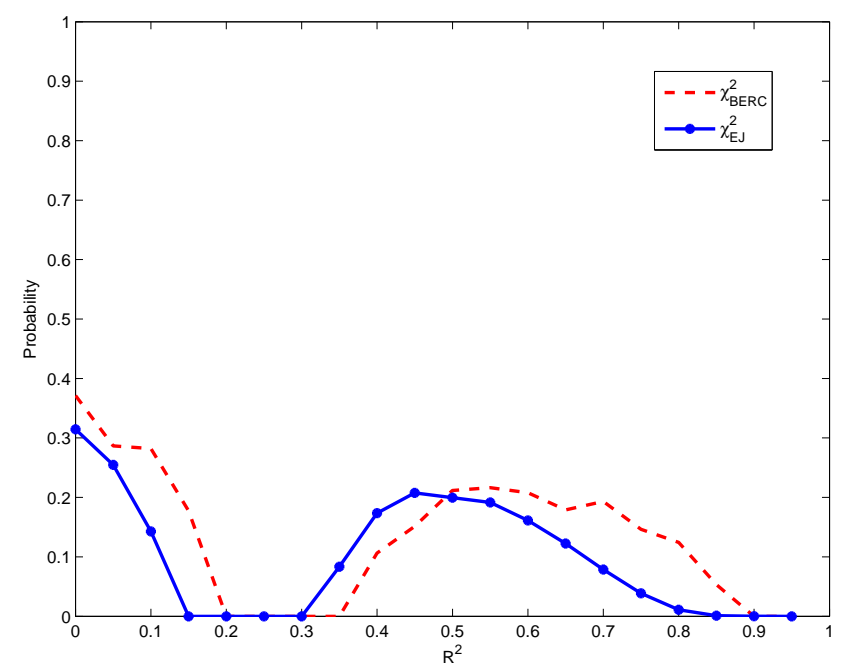

The probability $q$, with which a pretest using the underlying tests $\left(t_{\gamma}^{\mathrm{ADF}}\right.$ and $\lambda_{\max }$ for $\tilde{\chi}_{\mathcal{I}}^{2}\left(t_{\gamma}^{\mathrm{ADF}}, \lambda_{\max }\right)$, denoted $\chi_{\mathrm{EJ}}^{2}$ in the plot; and analogously for $\hat{F}, t_{\gamma}^{\mathrm{ECR}}$ and $\chi_{\mathrm{BERC}}^{2}$ ) needs to select the weaker test for our Fisher test to be at least as powerful as the pretest, is plotted against $R^{2} . K-1=1$ and $c=-15$.

test. A pretest would therefore likely be less powerful than the strategies advocated here. Some calculations may help to illustrate this point. Let $q$ denote the probability that the inferior test is selected. As an example, consider from Table $3 \lambda_{\max }, t_{\gamma}^{\mathrm{ADF}}$ and $\tilde{\chi}_{\mathcal{I}}^{2}\left(t_{\gamma}^{\mathrm{ADF}}, \lambda_{\max }\right)$ for $R^{2}=0.75$ and $c=-15$. A pretest, if available, would need to select the worse test ( $\left.t_{\gamma}^{\mathrm{ADF}}\right)$ in only $q=$ $(0.954-0.984) /(0.210-0.984) \times 100 \approx 4 \%$ of the cases for it to be inferior to $\tilde{\chi}_{\mathcal{I}}^{2}\left(t_{\gamma}^{\mathrm{ADF}}, \lambda_{\max }\right)$. For $\tilde{\chi}_{\mathcal{I}}^{2}\left(t_{\gamma}^{\mathrm{ADF}}, \lambda_{\max }\right), \tilde{\chi}_{\mathcal{I}}^{2}\left(t_{\gamma}^{\mathrm{ECR}}, \hat{F}\right), c=-15$ and $K-1=1$, Figure 7 plots $q$ against $R^{2}$ for that case. We see that $q$ never exceeds 0.3 , and even find $q=0$ for $R^{2} \in[0.15,0.3] \cup(0.85,1)$ (in the case of $\left.\tilde{\chi}_{\mathcal{I}}^{2}\left(t_{\gamma}^{\mathrm{ADF}}, \lambda_{\max }\right)\right)$, reflecting that $\tilde{\chi}_{\mathcal{I}}^{2}$ is sometimes as or more powerful than even a perfect pretest.

Remark 7. It is also tempting to develop ' $R^{2}$-weighted' versions of the meta tests. Consider e.g. $\tilde{\chi}_{\mathcal{I}, R^{2}}^{2}:=-2 \sum_{i \in \mathcal{I}} \varpi_{i}\left(R^{2}\right) \ln \left(p_{i}\right)$, where $\varpi_{i}$ is a weight function such that $\sum_{i \in \mathcal{I}} \varpi_{i}\left(R^{2}\right)=|\mathcal{I}|$ (in (6), each $i$ implicitly has $\varpi_{i}\left(R^{2}\right)=1$ ). Again, an estimator $\hat{R}^{2}$ would be necessary. Moreover, if the weights $\varpi_{i}$ depend on $R^{2}$, so would the null distribution of a weighted meta test like $\tilde{\chi}_{\mathcal{I}, R^{2}}^{2}$. Hence, $\tilde{\chi}_{\mathcal{I}, R^{2}}^{2}$ would no longer be nuisance-parameter free, making such an approach unattractive.

\section{Bootstrap Analogs}

The previous results rely entirely on asymptotic theory. The combination tests cannot be expected not to share small-sample deficiencies of the underlying cointegration tests. The small-sample behavior of cointegration tests has, among many others, been analyzed by Haug (1996), who finds the tests to be somewhat sensitive to short-run dynamics in the errors. In particular, the finitesample size of the tests depends on the choice of estimation method for these nuisance parameters. Thus, the local asymptotic power curves presented above are effectively approximations to the 
tests' finite-sample power curves. The bootstrap has recently been successfully employed to improve the small-sample behavior of cointegration tests (Swensen, 2006; Palm et al., 2009). We therefore now introduce bootstrap analogs of the combination tests to provide potentially more reliable inference in small samples. Recall the aggregator of $p$-values from the Fisher test,

$$
\tilde{\chi}_{\mathcal{I}}^{2}=-2 \sum_{i=1}^{|\mathcal{I}|} \ln \left(p_{i}\right) .
$$

To bootstrap the distribution of $\tilde{\chi}_{\mathcal{I}}^{2}$, we require a method to bootstrap cointegration tests. A suitable procedure has recently been proposed by Swensen (2006). In brief, Swensen's procedure resamples residuals from an estimated VECM representation of the data-generating process (DGP) to then generate integrated but non-cointegrated time series. We propose the following Algorithm to estimate the finite-sample distribution of $\tilde{\chi}_{\mathcal{I}}^{2}$.

\section{Algorithm 1.}

1. Estimate the unrestricted VAR

$$
\boldsymbol{z}_{t}=\sum_{p=1}^{P} \boldsymbol{\Phi}_{p} \boldsymbol{z}_{t-p}+\boldsymbol{d}_{t}+\boldsymbol{\varepsilon}_{t}
$$

to obtain estimates $\hat{\boldsymbol{d}}_{t}, \hat{\boldsymbol{\Phi}}_{p}$ and residuals $\hat{\boldsymbol{\varepsilon}}_{t}$. Transform $\hat{\boldsymbol{\Phi}}_{p}, p=1, \ldots, P$, to $\hat{\boldsymbol{\Gamma}}_{p}, p=$ $1, \ldots, P-1$, as in representation (3). ${ }^{7}$

2. Check that the system has no explosive root, i.e. $\|z\|>1$, by solving $\operatorname{det}\{\hat{\boldsymbol{B}}(z)\}=0$, where

$$
\hat{\boldsymbol{B}}(z):=\boldsymbol{I}_{K}-\hat{\boldsymbol{\Gamma}}_{1} z-\cdots-\hat{\boldsymbol{\Gamma}}_{P-1} z^{P-1} \cdot 8
$$

3. If so, draw $B$ series of pseudo errors $\left\{\varepsilon_{t, b}^{*}\right\}_{t=P, \ldots, T}^{b=1, \ldots, B}$ by resampling non-parametrically with replacement from the residuals $\left\{\hat{\varepsilon}_{t}\right\}_{t=P, \ldots, T}$.

4. With $\left\{\boldsymbol{\varepsilon}_{t, b}^{*}\right\}_{t=P, \ldots, T}^{b=1, \ldots, B}$, construct $B$ series of pseudo observations $\boldsymbol{z}_{t, b}^{*}$ from

$$
\Delta \boldsymbol{z}_{t, b}^{*}=\sum_{p=1}^{P-1} \hat{\boldsymbol{\Gamma}}_{p} \Delta \boldsymbol{z}_{t-p, b}^{*}+\hat{\boldsymbol{d}}_{t}+\boldsymbol{\varepsilon}_{t, b}^{*} .
$$

For the initial observations, set $\boldsymbol{z}_{t, b}^{*}=\boldsymbol{z}_{t}, t=0, \ldots, P-1 .^{9}$

5. Compute the vector of test statistics $\boldsymbol{\xi}_{b}^{*}:=\left(\xi_{1, b}^{*}, \ldots, \xi_{|\mathcal{I}|, b}^{*}\right)^{\prime}$, for each $b=1, \ldots, B$.

\footnotetext{
${ }^{7}$ See e.g. Hamilton (1994, Eq. 19.1.38) for the procedure. One could alternatively estimate a restricted VAR for $\Delta \boldsymbol{z}_{t}$, imposing the null of no cointegration (cf. Swensen, 2006). However, as Paparoditis and Politis (2003) show for unit-root tests, imposing such a restriction may lead to a power loss.

${ }^{8}$ See Swensen (2006, Remark 1) and Johansen (1995, p. 71) for a discussion of this technical requirement. Note that under $h=0, \hat{\boldsymbol{\alpha}} \hat{\boldsymbol{\beta}}^{\prime}=\mathbf{0}$ in Swensen's notation, such that we have $\hat{A}(z)=(1-z) \hat{\boldsymbol{B}}(z)$, with the l.h.s. in Swensen's notation again. Thus his condition (iii) is equivalent to (16) in our context.

${ }^{9}$ Since we require pseudo observations that are integrated but non-cointegrated, $\boldsymbol{\Pi}=\mathbf{0}$ is imposed.
} 
6. Estimate the distribution function of the test statistic of each test as

$$
\#\left\{\xi_{i, h}^{*} \leq x \mid h=1, \ldots, B\right\} / B=: 1-\Xi_{i}^{*}(x)
$$

and calculate the corresponding p-values $p_{i, b}^{*}:=\Xi_{i}^{*}\left(\xi_{i, b}^{*}\right)$.Correspondingly, calculate the $p$ values of the test statistics $\xi_{i}$ on the original data $\boldsymbol{z}_{i, t}$ by $p_{i}^{*}:=\Xi_{i}^{*}\left(\xi_{i}\right)$.

7. Obtain the corresponding aggregate $\tilde{\chi}_{\mathcal{I}}^{2}$ test statistic

$$
\tilde{\chi}_{\mathcal{I}, b}^{2, *}=-2 \sum_{i=1}^{|\mathcal{I}|} \ln \left(p_{i, b}^{*}\right) .
$$

8. Estimate the cumulative distribution function $F_{\mathcal{F}_{\mathcal{I}}^{*}}$ of the $\tilde{\chi}_{\mathcal{I}, b}^{2, *}$ by

$$
\hat{F}_{\mathcal{F}_{\mathcal{I}}^{*}}(x):=\#\left\{\tilde{\chi}_{\mathcal{I}, h}^{2, *} \leq x \mid h=1, \ldots, B\right\} / B
$$

This provides us with a bootstrap version of the $\tilde{\chi}_{\mathcal{I}}^{2}$ test,

$$
\tilde{\chi}_{\mathcal{I}}^{2, *}=-2 \sum_{i=1}^{|\mathcal{I}|} \ln \left(p_{i}^{*}\right),
$$

where we reject $\mathcal{H}_{0}$ at level $\alpha$ if $\tilde{\chi}_{\mathcal{I}}^{2, *}$ exceeds the $(1-\alpha)$-quantile of $\hat{F}_{\mathcal{F}_{\mathcal{I}}^{*}}$.

Heuristically, the method can be expected to work as follows. Swensen (2006) analytically proves that his bootstrap procedure (i.e. steps 1-4 in Algorithm 1) yield pseudo-observations $\boldsymbol{z}_{t, b}^{*}$ which have a representation asymptotically equivalent to the true DGP. Moreover, he proves that steps 5 and 6 consistently estimate the null distribution of the Johansen $\lambda_{\text {trace }}$ test, hence yielding consistent estimates of $p$-values. Therefore, we can expect the proposition to carry over to the cointegration tests mentioned above, as these essentially also rely on the availability of suitable $\boldsymbol{z}_{t, b}^{*}$. The CMT with $\boldsymbol{\xi}:=\left(\xi_{1}, \ldots, \xi_{|\mathcal{I}|}\right)^{\prime}$ as functions of the observations $\boldsymbol{z}_{i, t}$, for which an invariance principle holds, ensures a well-defined joint distribution of the statistics $\boldsymbol{\xi}$. That joint distribution can be consistently estimated with Algorithm 1 under fairly weak regularity conditions (Horowitz, 2001). We provide extensive numerical support for this argument in Section $6{ }^{10}$

Remark 8. Algorithm 1 is only about as computationally demanding as Swensen's (2006). It requires resampling the same number of pseudo-observations, and no double bootstrapping. The difference to Swensen's algorithm is that $|\mathcal{I}|$ instead of one statistic $\left(\lambda_{\text {trace }}\right)$ need to be calculated for each $b$.

Remark 9. In view of the equivalence of the $U R_{\psi_{\mathcal{I}}}$ and min-test established in Remark 4, a version of Algorithm 1 also provides bootstrap $U R_{\psi_{\mathcal{I}}}$ tests by bootstrapping the distribution of $\min _{i \in \mathcal{I}} p_{i}$. We reject $\mathcal{H}_{0}$ if $\min _{i \in \mathcal{I}} p_{i}<\hat{F}_{\text {min }}^{*,-1}(\alpha)$, the $\alpha$-quantile of the bootstrap distribution $\hat{F}_{\text {min }}^{*}$.

\footnotetext{
${ }^{10}$ Appendix $\mathrm{C}$ describes an alternative bootstrap test that we found to have slightly higher power in unreported simulations. As that approach requires stronger theoretical assumptions, we advocate using $\tilde{\chi}_{\mathcal{I}}^{2, *}$.
} 


\section{Monte Carlo Experiments}

\subsection{Setup}

We now study the finite-sample properties of the tests in a series of Monte Carlo experiments. As shown above, different tests for cointegration differ in their power against different points in the $\left(c-R^{2}\right)$-space of the alternative hypothesis. Further, e.g. Johansen's $\lambda_{\max }$ test can be expected to be relatively more powerful if $\Delta \boldsymbol{z}_{t}$ is indeed generated by a finite order VECM. Since our tests combine information from tests that are powerful in different directions, a likely advantage of our testing strategy is more robust power across different DGPs. We consider the following DGPs:

$$
\begin{array}{ll}
\operatorname{DGP}(\mathrm{A}): & \Delta x_{t}=v_{1 t} \\
& y_{t}=\theta x_{t}+u_{t} \\
& u_{t}=\rho_{T} u_{t-1}+v_{2 t}
\end{array}
$$

where $\theta=1$. The autoregressive coefficient $\rho_{T}=1+c / T$. $\mathcal{H}_{0}$ is obtained when $c=0$, whereas we parameterize $\mathcal{H}_{1}$ by $c=-15 .{ }^{11}$ The errors $\boldsymbol{v}_{t}$ are drawn from

$$
\boldsymbol{v}_{t}=\left(\begin{array}{c}
v_{1 t} \\
v_{2 t}
\end{array}\right) \stackrel{i i d}{\sim} \mathcal{N}(\mathbf{0}, \boldsymbol{\Omega}), \quad \text { where } \quad \boldsymbol{\Omega}=\left(\begin{array}{ll}
1 & \delta \\
\delta & 1
\end{array}\right)
$$

For $R^{2}=\delta^{2}$, we select $R^{2}=\{0,0.25,0.5,0.75\}$. DGP(A) closely follows Pesavento's model (1). To investigate the generality of her setup we additionally investigate the following DGPs.

$$
\begin{array}{ll}
\operatorname{DGP}(\mathrm{B}): & \Delta \boldsymbol{z}_{t}=\boldsymbol{\Pi} \boldsymbol{z}_{t-1}+\boldsymbol{\Gamma} \Delta \boldsymbol{z}_{t-1}+\boldsymbol{u}_{t}, \quad \text { where } \quad \boldsymbol{\Gamma}=0.2 \boldsymbol{I}_{2} \\
\operatorname{DGP}(\mathrm{C}): & y_{t}+\eta x_{t}=a_{1 t}, \quad y_{t}+\theta x_{t}=a_{2 t}, \quad \text { where } \quad \theta=-1, \eta=-1 / 2 \quad \text { and } \\
& a_{1 t}=a_{1 t-1}+u_{1 t}, a_{2 t}=\rho_{T} a_{2 t-1}+u_{2 t} .
\end{array}
$$

In DGPs (B) and (C) we set $\boldsymbol{u}_{t}=\left(u_{1 t}, u_{2 t}\right) \stackrel{\text { iid }}{\sim} \mathcal{N}\left(\mathbf{0}, \boldsymbol{I}_{2}\right)$. For $(\mathrm{B}) \mathcal{H}_{0}$ is obtained when $\boldsymbol{\Pi}=\mathbf{0}$, whereas we parameterize $\mathcal{H}_{1}$ by $\boldsymbol{\Pi}=\left(\begin{array}{ll}1 & 0\end{array}\right)^{\prime}(.15-.15)$. For $(\mathrm{C}), \mathcal{H}_{0}$ and $\mathcal{H}_{1}$ are parameterized as in (A). ${ }^{12}$ DGPs (A) and (C) are local, such that power ought to remain roughly constant when increasing $T$, while power should increase for DGP(B). These designs are widely used in Monte Carlo studies of cointegration tests. See e.g. Pesavento (2004, 2007) for (A), Swensen (2006) for (B), or Engle and Granger (1987), Haug (1996) and Gregory et al. (2004) for (C).

For each DGP, we draw 5,000 replications under $\mathcal{H}_{0}$ and $\mathcal{H}_{1}$. We choose $T \in\{50,75,100,150,200\}$. These time-series lengths correspond to typical sample sizes encountered in applied macroeconometric work, e.g. when using quarterly data. To mitigate the effect of initial conditions under $\mathcal{H}_{1}$, we simulate each DGP for $T+30$ time periods and discard the first 30 observations. For each

\footnotetext{
${ }^{11}$ Power results for other $c$ are given in Appendix D.

${ }^{12}$ Of course, Granger's representation theorem would allow us to write DGP(C) in a VECM form. However, error terms would be correlated, the matrix $\boldsymbol{\Pi}$ would have no rows of zeros under $\mathcal{H}_{1}$ and $\boldsymbol{\Gamma}$ would equal $\mathbf{0}$.
} 
replication, we compute the $U R^{*}$ and the $\tilde{\chi}_{\mathcal{I}}^{2, *}$ tests based on $B=10,000$ resamples. To keep the setup simple, we initially combine $|\mathcal{I}|=2$ underlying tests. In particular, we select Johansen's (1988) $\lambda_{\max }$ test and Engle and Granger's (1987) $t_{\gamma}^{\mathrm{ADF}}$ test. We opt for these tests as they are widely used in applied work. Moreover, Section 4 establishes that these tests have high power for different values of the nuisance parameter $R^{2}$, such that combining them seems promising. For comparison, we also combine Boswijk's (1994) $\hat{F}$ test and Banerjee et al.'s (1998) $t_{\gamma}^{\mathrm{ECR}}$ test.

To investigate the relative performance of the new tests, we compare them to the following cointegration tests: First, the standard $t_{\gamma}^{\mathrm{ADF}}, \lambda_{\max }, t_{\gamma}^{\mathrm{ECR}}$ and $\hat{F}$ tests, where we reject $\mathcal{H}_{0}$ if the test statistics exceed the asymptotic level- $\alpha$ critical value. ${ }^{13}$ Second, we investigate bootstrap versions of the tests (denoted in the following by $t_{\gamma}^{\mathrm{ADF}, *}, \lambda_{\mathrm{max}}^{*}, t_{\gamma}^{\mathrm{ECR}, *}$ and $\hat{F}^{*}$ ), which are byproducts of our $U R^{*}$ and $\tilde{\chi}_{\mathcal{I}}^{2, *}$ tests. Third, we compute a 'naive' meta test that rejects whenever at least one of a set of single tests rejects. We call this test 'naive' because it ignores the multipletesting nature of the problem. This test reveals the size distortion incurred by selecting the most rejective from a set of cointegration tests.

Implementation of the cointegration tests requires to select an order $\hat{P}$ of lagged differences to account for auto-correlation. In practice this is often done via some lag-length selection criterion, see e.g. Lütkepohl (2005). To reduce the computational burden we waive this option and use the correct lag order throughout. All tests are based on case (iii).

\subsection{Results}

Table 4 reports the small sample size of the tests based on $\lambda_{\max }$ and $t_{\gamma}^{\mathrm{ADF}}$ at the $5 \%$ level. Results for DGP(A) are based on $R^{2}=0.25 .^{14}$ As expected, the 'naive' test is oversized and its size exceeds that of the single tests by approximately 3 - 4 percentage points. ${ }^{15}$ All other tests control size reasonably well. The $U R_{\psi_{\mathcal{I}}}$ test (and to a lesser extent also the $\tilde{\chi}_{\mathcal{I}}^{2}$ test) exhibits a slight upward size distortion for small $T$, partly due to a distortion of $t_{\gamma}^{\mathrm{ADF}}$ for small $T$. However, this size distortion vanishes for $T \geqslant 100$. The bootstrap versions of the tests seem to approach the nominal size somewhat more quickly, which reflects that the bootstrap distribution generated in Algorithm 1 generally is a somewhat more accurate approximation to the unknown-finite sample distribution than the asymptotic one.

Table 5 reports the small sample power of the $\lambda_{\max }$ and $t_{\gamma}^{\mathrm{ADF}}$-based tests at the level $\alpha$ of $5 \%$. For DGP(A), we find that the local asymptotic results from Section 4 predict the finite-sample results rather well, in that $t_{\gamma}^{\mathrm{ADF}}$ and $\lambda_{\max }$ again have similar power for this $R^{2}$. Moreover, the combination tests $\tilde{\chi}_{\mathcal{I}}^{2}$ and $U R_{\psi_{\mathcal{I}}}$ again outperform both single tests. As expected, power increases in $T$ for all tests for DGP(B). While of the single tests the $t_{\gamma}^{\mathrm{ADF}}$ test is the most powerful single test for DGP(C), the $\lambda_{\max }$ and $\lambda_{\max }^{*}$ tests are most powerful for DGP(B). This result may not

\footnotetext{
${ }^{13}$ In the case of the $t_{\gamma}^{\mathrm{ADF}}$ test we follow the standard practice of using MacKinnon (1996)-type critical values.

${ }^{14}$ Appendix D reports results for other values of $R^{2}$. Furthermore, we ran all simulations at the $1 \%$ and $10 \%$ level. We also experimented with a version of $\operatorname{DGP}(\mathrm{C})$ with $\operatorname{AR}(1)$ error terms instead of white noise $\boldsymbol{u}_{t}$. All results are qualitatively similar; additional results are available upon request.

${ }^{15}$ This size distortion is very close to the one that can be inferred from Table I in Gregory et al. (2004).
} 
Table 4: Small-sample size based on $\lambda_{\max }$ and $t_{\gamma}^{\mathrm{ADF}}$

\begin{tabular}{|c|c|c|c|c|c|c|c|c|c|c|c|}
\hline \multirow[b]{2}{*}{ DGP } & \multirow[b]{2}{*}{$T$} & \multicolumn{5}{|c|}{ Bootstrap tests } & \multicolumn{5}{|c|}{ asymptotic tests } \\
\hline & & $\lambda_{\max }^{*}$ & $t_{\gamma}^{\mathrm{ADF}, *}$ & naive* & $\tilde{\chi}_{\mathcal{I}}^{2, *}$ & $U R_{\psi_{\mathcal{I}}}^{*}$ & $\lambda_{\max }$ & $t_{\gamma}^{\mathrm{ADF}}$ & naive & $\tilde{\chi}_{\mathcal{I}}^{2}$ & $U R_{\psi_{\mathcal{I}}}$ \\
\hline \multirow[t]{5}{*}{ (A) } & 50 & 0.051 & 0.048 & 0.078 & 0.051 & 0.048 & 0.054 & 0.080 & 0.113 & 0.062 & 0.084 \\
\hline & 75 & 0.044 & 0.042 & 0.072 & 0.042 & 0.040 & 0.055 & 0.077 & 0.110 & 0.059 & 0.080 \\
\hline & 100 & 0.048 & 0.048 & 0.076 & 0.049 & 0.046 & 0.054 & 0.075 & 0.111 & 0.056 & 0.072 \\
\hline & 150 & 0.046 & 0.046 & 0.079 & 0.045 & 0.048 & 0.054 & 0.063 & 0.099 & 0.049 & 0.069 \\
\hline & 200 & 0.055 & 0.050 & 0.086 & 0.059 & 0.057 & 0.048 & 0.058 & 0.090 & 0.047 & 0.059 \\
\hline \multirow[t]{5}{*}{ (B) } & 50 & 0.052 & 0.050 & 0.080 & 0.051 & 0.049 & 0.067 & 0.069 & 0.108 & 0.063 & 0.077 \\
\hline & 75 & 0.050 & 0.050 & 0.078 & 0.049 & 0.047 & 0.060 & 0.062 & 0.098 & 0.060 & 0.065 \\
\hline & 100 & 0.047 & 0.045 & 0.075 & 0.046 & 0.046 & 0.061 & 0.059 & 0.093 & 0.060 & 0.066 \\
\hline & 150 & 0.050 & 0.047 & 0.073 & 0.050 & 0.046 & 0.057 & 0.060 & 0.090 & 0.057 & 0.061 \\
\hline & 200 & 0.050 & 0.055 & 0.081 & 0.053 & 0.057 & 0.057 & 0.063 & 0.092 & 0.063 & 0.063 \\
\hline \multirow[t]{5}{*}{ (C) } & 50 & 0.045 & 0.054 & 0.083 & 0.050 & 0.048 & 0.053 & 0.081 & 0.114 & 0.060 & 0.081 \\
\hline & 75 & 0.044 & 0.043 & 0.073 & 0.041 & 0.041 & 0.055 & 0.076 & 0.110 & 0.055 & 0.077 \\
\hline & 100 & 0.046 & 0.051 & 0.082 & 0.048 & 0.049 & 0.054 & 0.069 & 0.103 & 0.054 & 0.072 \\
\hline & 150 & 0.048 & 0.050 & 0.082 & 0.047 & 0.048 & 0.054 & 0.064 & 0.099 & 0.049 & 0.070 \\
\hline & 200 & 0.055 & 0.051 & 0.088 & 0.059 & 0.055 & 0.048 & 0.058 & 0.089 & 0.044 & 0.060 \\
\hline
\end{tabular}

Average rejection rates at nominal level of 5\%. 5,000 replications and 10,000 bootstrap replications. $t_{\gamma}^{\mathrm{ADF}}$ and $\lambda_{\max }$ refer to Engle and Granger (1987) and Johansen (1988) tests, $t_{\gamma}^{\mathrm{ADF}, *}$ and $\lambda_{\max }^{*}$ are their bootstrap counterparts. naive rejects when $t_{\gamma}^{\mathrm{ADF}, *}$ or $\lambda_{\max }^{*}$ or both reject. $U R_{\psi_{\mathcal{I}}}$ is the test defined by (9) and (11) and and $U R^{*}$ is the bootstrap counterpart. $\tilde{\chi}_{\mathcal{I}}^{2}$ is the Fisher test (6) and $\tilde{\chi}_{\mathcal{I}}^{2, *}$ is its bootstrap counterpart. ( $U R^{*}$ and $\tilde{\chi}_{\mathcal{I}}^{2, *}$ are described in Algorithm 1.)

be entirely surprising, as both tests were originally designed having DGPs of type (B) and (C) respectively in mind. For those DGPs, $\tilde{\chi}_{\mathcal{I}}^{2}$ and $U R_{\psi_{\mathcal{I}}}$ again both perform similarly and well, in that their power is again close or superior to that of the better of the two constituent tests. The (size-adjusted) power of the bootstrap versions is very similar to that of the asymptotic tests throughout.

Tables 6 and 7 reports analogous results for the tests based on $\hat{F}$ and $t_{\gamma}^{\mathrm{ECR}}$. Once more, all tests have a slight upward size distortion for small $T$, which vanishes as $T$ increases. The performance of the single $\hat{F}$ and $t_{\gamma}^{\mathrm{ECR}}$ tests is again similar, as predicted by Section 4 . It is therefore not surprising that the performance of the meta tests $\tilde{\chi}_{\mathcal{I}}^{2}$ and $U R_{\psi_{\mathcal{I}}}$ is also very similar to that of the single tests. Comparing Tables 5 and 7 , we find that $t_{\gamma}^{\mathrm{ADF}}$ and $\lambda_{\max }$ outperform either $\hat{F}$ or $t_{\gamma}^{\mathrm{ECR}}$ for DGP(C) and (B), respectively, which again reflects that the former tests were designed having such DGPs in mind. This also implies that the superior local asymptotic power properties of $\hat{F}$ and $t_{\gamma}^{\mathrm{ECR}}$ found by Pesavento (2004) may be somewhat model-specific, in that these results do not carry over to other parameterizations of cointegrated systems such as DGPs (B) and (C). Hence, it would be premature to recommend routine application of either the $\hat{F}$ or $t_{\gamma}^{\mathrm{ECR}}$ test in practice. Indeed, our meta tests are attractive because they not only offer a robust insurance against wrong test choice given the nuisance parameter $R^{2}$, but effectively also robustness when there is uncertainty over the form of the DGP, as is the case in practice. 
Table 5: Small-sample power based on $\lambda_{\max }$ and $t_{\gamma}^{\mathrm{ADF}}$

\begin{tabular}{|c|c|c|c|c|c|c|c|c|c|c|c|}
\hline \multirow[b]{2}{*}{ DGP } & \multirow[b]{2}{*}{$T$} & \multicolumn{5}{|c|}{ Bootstrap tests } & \multicolumn{5}{|c|}{ asymptotic tests } \\
\hline & & $\lambda_{\max }^{*}$ & $t_{\gamma}^{\mathrm{ADF}, *}$ & naive* & $\tilde{\chi}_{\mathcal{I}}^{2, *}$ & $U R_{\psi_{\mathcal{I}}}^{*}$ & $\lambda_{\max }$ & $t_{\gamma}^{\mathrm{ADF}}$ & naive & $\tilde{\chi}_{\mathcal{I}}^{2}$ & $U R_{\psi_{\mathcal{I}}}$ \\
\hline \multirow[t]{5}{*}{ (A) } & 50 & 0.284 & 0.255 & 0.389 & 0.337 & 0.273 & 0.288 & 0.362 & 0.462 & 0.359 & 0.374 \\
\hline & 75 & 0.281 & 0.246 & 0.381 & 0.324 & 0.264 & 0.290 & 0.320 & 0.440 & 0.343 & 0.344 \\
\hline & 100 & 0.269 & 0.239 & 0.368 & 0.317 & 0.259 & 0.279 & 0.296 & 0.413 & 0.307 & 0.318 \\
\hline & 150 & 0.265 & 0.235 & 0.366 & 0.310 & 0.252 & 0.279 & 0.270 & 0.394 & 0.301 & 0.302 \\
\hline & 200 & 0.274 & 0.233 & 0.361 & 0.306 & 0.257 & 0.275 & 0.258 & 0.386 & 0.284 & 0.290 \\
\hline \multirow[t]{5}{*}{ (B) } & 50 & 0.081 & 0.081 & 0.128 & 0.082 & 0.079 & 0.099 & 0.104 & 0.161 & 0.100 & 0.118 \\
\hline & 75 & 0.142 & 0.114 & 0.204 & 0.132 & 0.138 & 0.170 & 0.143 & 0.238 & 0.157 & 0.172 \\
\hline & 100 & 0.265 & 0.174 & 0.330 & 0.240 & 0.243 & 0.293 & 0.211 & 0.372 & 0.269 & 0.283 \\
\hline & 150 & 0.598 & 0.379 & 0.663 & 0.573 & 0.561 & 0.623 & 0.402 & 0.691 & 0.591 & 0.593 \\
\hline & 200 & 0.877 & 0.625 & 0.908 & 0.871 & 0.859 & 0.888 & 0.646 & 0.918 & 0.880 & 0.869 \\
\hline \multirow[t]{5}{*}{ (C) } & 50 & 0.179 & 0.271 & 0.321 & 0.278 & 0.223 & 0.194 & 0.372 & 0.413 & 0.310 & 0.329 \\
\hline & 75 & 0.170 & 0.258 & 0.304 & 0.259 & 0.206 & 0.193 & 0.342 & 0.384 & 0.285 & 0.297 \\
\hline & 100 & 0.171 & 0.271 & 0.319 & 0.276 & 0.215 & 0.177 & 0.316 & 0.358 & 0.268 & 0.271 \\
\hline & 150 & 0.160 & 0.252 & 0.297 & 0.255 & 0.202 & 0.178 & 0.299 & 0.344 & 0.258 & 0.260 \\
\hline & 200 & 0.178 & 0.256 & 0.303 & 0.263 & 0.210 & 0.173 & 0.277 & 0.327 & 0.239 & 0.246 \\
\hline
\end{tabular}

See notes to Table 4. For DGP(A), $R^{2}=0.25$ and for $(\mathrm{A})$ and $(\mathrm{C}), c=-15$.

\subsection{Extension to more than two tests}

For expositional clarity we so far analyzed combinations of only $|\mathcal{I}|=2$ tests, combining the $t_{\gamma}^{\mathrm{ADF}}$ and $\lambda_{\max }$ or the $\hat{F}$ and $t_{\gamma}^{\mathrm{ECR}}$ tests to illustrate our approach. Of course, as discussed in Section 3 , our approach can accommodate other and more tests as well. Potentially, this yields further gains in power if the additional tests have high power for the given nuisance parameter value.

We therefore now combine all four tests considered in the previous subsection (denoted $\tilde{\chi}_{\mathcal{I}}^{2}(4)$ ) and compare its performance to the combination tests based on $\lambda_{\max }$ and $t_{\gamma}^{\mathrm{ADF}}$, denoted $\tilde{\chi}_{\mathcal{I}}^{2}(2)$. In view of the qualitatively similar performance of bootstrap and asymptotic tests we focus on the latter for brevity. We find that the more general $\tilde{\chi}_{\mathcal{I}}^{2}(4)$ test outperforms its simple counterpart $\tilde{\chi}_{\mathcal{I}}^{2}(2)$ rather markedly. Of course, the asymptotic results from Section 4 predict that this is a setting where $t_{\gamma}^{\mathrm{ADF}}$ and $\lambda_{\max }$ are less powerful than $\hat{F}$ and $t_{\gamma}^{\mathrm{ECR}}$, such that one might want to choose the latter only. Yet, bearing Remark 6 in mind, such knowledge about the DGP will rarely be available in practice. Indeed, we view it as implausible that researchers should feel the need to conduct statistical inference about a key feature of the time series at hand - cointegration versus non-cointegration - while at the same time having accurate knowledge about some nuisance parameter. Hence, the extra robustness that can be gained from combining $|\mathcal{I}|=4$ tests may well be attractive for practitioners.

To summarize, both $U R_{\psi_{\mathcal{I}}}$ and $\tilde{\chi}_{\mathcal{I}}^{2}$ control the size of the test and yet provide a robust, powerful and flexible alternative to traditional cointegration tests. 
Table 6: Small-sample size based on $\hat{F}$ and $t_{\gamma}^{\mathrm{ECR}}$

\begin{tabular}{|c|c|c|c|c|c|c|c|c|c|c|c|}
\hline \multirow[b]{2}{*}{ DGP } & \multirow[b]{2}{*}{$T$} & \multicolumn{5}{|c|}{ Bootstrap tests } & \multicolumn{5}{|c|}{ asymptotic tests } \\
\hline & & $\hat{F}^{*}$ & $t_{\gamma}^{\mathrm{ECR}, *}$ & naive* & $\tilde{\chi}_{\mathcal{I}}^{2, *}$ & $U R_{\psi_{\mathcal{I}}}^{*}$ & $\hat{F}$ & $t_{\gamma}^{\mathrm{ECR}}$ & naive & $\tilde{\chi}_{\mathcal{I}}^{2}$ & $U R_{\psi_{\mathcal{I}}}$ \\
\hline \multirow[t]{5}{*}{ (A) } & 50 & 0.050 & 0.051 & 0.062 & 0.051 & 0.052 & 0.084 & 0.077 & 0.093 & 0.079 & 0.082 \\
\hline & 75 & 0.047 & 0.045 & 0.055 & 0.045 & 0.046 & 0.076 & 0.072 & 0.086 & 0.075 & 0.076 \\
\hline & 100 & 0.050 & 0.053 & 0.061 & 0.051 & 0.052 & 0.073 & 0.073 & 0.084 & 0.074 & 0.073 \\
\hline & 150 & 0.045 & 0.045 & 0.053 & 0.045 & 0.044 & 0.065 & 0.062 & 0.073 & 0.065 & 0.066 \\
\hline & 200 & 0.052 & 0.055 & 0.062 & 0.054 & 0.052 & 0.057 & 0.053 & 0.063 & 0.054 & 0.057 \\
\hline \multirow[t]{5}{*}{ (B) } & 50 & 0.050 & 0.056 & 0.064 & 0.054 & 0.053 & 0.069 & 0.068 & 0.079 & 0.070 & 0.069 \\
\hline & 75 & 0.051 & 0.050 & 0.060 & 0.050 & 0.052 & 0.067 & 0.064 & 0.076 & 0.065 & 0.065 \\
\hline & 100 & 0.044 & 0.044 & 0.052 & 0.044 & 0.044 & 0.063 & 0.060 & 0.072 & 0.061 & 0.063 \\
\hline & 150 & 0.049 & 0.047 & 0.058 & 0.049 & 0.050 & 0.060 & 0.057 & 0.069 & 0.058 & 0.058 \\
\hline & 200 & 0.054 & 0.057 & 0.066 & 0.056 & 0.055 & 0.064 & 0.063 & 0.071 & 0.062 & 0.063 \\
\hline \multirow[t]{5}{*}{ (C) } & 50 & 0.049 & 0.054 & 0.061 & 0.052 & 0.052 & 0.083 & 0.076 & 0.091 & 0.079 & 0.082 \\
\hline & 75 & 0.042 & 0.044 & 0.052 & 0.044 & 0.044 & 0.071 & 0.069 & 0.081 & 0.070 & 0.070 \\
\hline & 100 & 0.051 & 0.052 & 0.061 & 0.051 & 0.051 & 0.068 & 0.064 & 0.075 & 0.067 & 0.067 \\
\hline & 150 & 0.047 & 0.048 & 0.055 & 0.047 & 0.047 & 0.068 & 0.065 & 0.076 & 0.068 & 0.067 \\
\hline & 200 & 0.051 & 0.053 & 0.061 & 0.053 & 0.052 & 0.057 & 0.058 & 0.066 & 0.058 & 0.059 \\
\hline
\end{tabular}

See notes to Table 4. $\hat{F}$ and $t_{\gamma}^{\mathrm{ECR}}$ are from Boswijk (1994) and Banerjee et al. (1998). Starred tests are bootstrap counterparts.

\section{Empirical Application}

\subsection{Setup}

Naturally we are interested in the practical applicability and relevance of our approach. To shed light on this question, we revisit the studies which Gregory et al. (2004) investigated for 'mixed signals', i.e. conflicting cointegration test results. Gregory et al. (2004) analyze 34 studies which were published in the Journal of Applied Econometrics from 1994 to March/April 2001. ${ }^{16}$ From these studies we construct 159 data sets in which we test for cointegration. The data sets exhibit large differences in sample size $T$, which ranges from 27 to 7693 with a median size of 73 . Similarly the number of variables $K$ differs across studies and ranges from 2 to 11 .

Our goal is to document the extent to which conflicting test results arise in actual applications and how our proposed meta tests are able to heal this problem. As Gregory et al. (2004), we do not intend to suggest that the authors of the studies have been in any way strategic in their choice of which cointegration test to report. Most applied researchers tend to view the different tests as rather interchangeable, with the choice more dependent on the nature of the investigation.

We follow Gregory et al. (2004) closely in their setup. The original published studies employ different methods to test their specifications. To make the results comparable, we impose a unifying but standard methodology. If a test requires a dependent variable $y_{t}$, we follow the choice in the original paper if possible. If there is no obvious $y_{t}$, we choose it based on the highest coefficient of determination of first-stage regressions. We also need to allow for variation

\footnotetext{
${ }^{16}$ The raw data are available at http://qed.econ.queensu.ca/jae/2004-v19.1/gregory-haug-lomuto/. Our modified data sets are available upon request.
} 
Table 7: Small-sample power based on $\hat{F}$ and $t_{\gamma}^{\mathrm{ECR}}$

\begin{tabular}{|c|c|c|c|c|c|c|c|c|c|c|c|}
\hline \multirow[b]{2}{*}{ DGP } & \multirow[b]{2}{*}{$T$} & \multicolumn{5}{|c|}{ Bootstrap tests } & \multicolumn{5}{|c|}{ asymptotic tests } \\
\hline & & $\hat{F}^{*}$ & $t_{\gamma}^{\mathrm{ECR}, *}$ & naive* & $\tilde{\chi}_{\mathcal{I}}^{2, *}$ & $U R_{\psi_{\mathcal{I}}}^{*}$ & $\hat{F}$ & $t_{\gamma}^{\mathrm{ECR}}$ & naive & $\tilde{\chi}_{\mathcal{I}}^{2}$ & $U R_{\psi_{\mathcal{I}}}$ \\
\hline \multirow[t]{5}{*}{ (A) } & 50 & 0.433 & 0.415 & 0.467 & 0.431 & 0.426 & 0.553 & 0.517 & 0.578 & 0.542 & 0.542 \\
\hline & 75 & 0.433 & 0.409 & 0.464 & 0.427 & 0.426 & 0.528 & 0.491 & 0.553 & 0.517 & 0.519 \\
\hline & 100 & 0.423 & 0.400 & 0.452 & 0.418 & 0.417 & 0.496 & 0.463 & 0.526 & 0.487 & 0.488 \\
\hline & 150 & 0.419 & 0.392 & 0.450 & 0.413 & 0.413 & 0.474 & 0.435 & 0.500 & 0.463 & 0.463 \\
\hline & 200 & 0.422 & 0.387 & 0.448 & 0.409 & 0.411 & 0.457 & 0.413 & 0.478 & 0.440 & 0.445 \\
\hline \multirow[t]{5}{*}{ (B) } & 50 & 0.098 & 0.094 & 0.115 & 0.096 & 0.097 & 0.133 & 0.116 & 0.146 & 0.122 & 0.129 \\
\hline & 75 & 0.150 & 0.131 & 0.168 & 0.141 & 0.144 & 0.193 & 0.157 & 0.207 & 0.176 & 0.186 \\
\hline & 100 & 0.219 & 0.187 & 0.238 & 0.205 & 0.209 & 0.265 & 0.223 & 0.281 & 0.244 & 0.256 \\
\hline & 150 & 0.423 & 0.364 & 0.440 & 0.399 & 0.402 & 0.460 & 0.389 & 0.472 & 0.424 & 0.443 \\
\hline & 200 & 0.635 & 0.552 & 0.645 & 0.599 & 0.610 & 0.660 & 0.572 & 0.671 & 0.621 & 0.636 \\
\hline \multirow[t]{5}{*}{ (C) } & 50 & 0.217 & 0.247 & 0.255 & 0.237 & 0.227 & 0.297 & 0.321 & 0.336 & 0.315 & 0.306 \\
\hline & 75 & 0.210 & 0.234 & 0.244 & 0.226 & 0.217 & 0.281 & 0.300 & 0.313 & 0.294 & 0.288 \\
\hline & 100 & 0.216 & 0.245 & 0.255 & 0.237 & 0.226 & 0.254 & 0.278 & 0.290 & 0.272 & 0.261 \\
\hline & 150 & 0.203 & 0.227 & 0.235 & 0.218 & 0.209 & 0.246 & 0.270 & 0.282 & 0.264 & 0.256 \\
\hline & 200 & 0.212 & 0.233 & 0.243 & 0.226 & 0.219 & 0.232 & 0.259 & 0.269 & 0.248 & 0.240 \\
\hline
\end{tabular}

See notes to Table 4. $\hat{F}$ and $t_{\gamma}^{\mathrm{ECR}}$ are from Boswijk (1994) and Banerjee et al. (1998). Starred tests are bootstrap counterparts. For $\operatorname{DGP}(\mathrm{A}), R^{2}=0.25$ and for $(\mathrm{A})$ and $(\mathrm{C}), c=-15$.

in lag lengths $\hat{P}$ across data sets. The literature discusses a number of different methods for choosing $\hat{P}$. We have chosen a fairly standard one and determine $\hat{P}$ using a Schwarz Information Criterion (BIC) as described e.g. in Lütkepohl (2005, Secs. 4.3.2 and 8.1). We search over the range $1 \leq \hat{P} \leq \min \left(8\left(\frac{T}{100}\right)^{1 / 5}, \frac{T-2}{2(K+2)}\right)$, and impose the same number of lags for all tests. Our qualitative conclusions would not be different if alternative selection methods were employed. All tests include a constant and a trend.

\subsection{Results}

We compare the results of applying $\lambda_{\max }, t_{\gamma}^{\mathrm{ADF}}, t_{\gamma}^{\mathrm{ECR}}$ and $\hat{F}$ as underlying single tests with the $U R_{\psi_{\mathcal{I}}}\left(\lambda_{\max }, t_{\gamma}^{\mathrm{ADF}}\right), U R_{\psi_{\mathcal{I}}}\left(t_{\gamma}^{\mathrm{ECR}}, \hat{F}\right), \tilde{\chi}_{\mathcal{I}}^{2}\left(\lambda_{\max }, t_{\gamma}^{\mathrm{ADF}}\right), \tilde{\chi}_{\mathcal{I}}^{2}\left(t_{\gamma}^{\mathrm{ECR}}, \hat{F}\right)$, and $\tilde{\chi}_{\mathcal{I}}^{2}\left(\lambda_{\max }, t_{\gamma}^{\mathrm{ADF}}, t_{\gamma}^{\mathrm{ECR}}, \hat{F}\right)$ tests. Specifically, we first check whether all single tests agree or not in their testing decision at the $5 \%$ level, see left panel of Table 9 . In those cases where conflicting test results occur we check what the test used in the original paper had suggested as a result (more precisely what would have been the outcome of our version with the chosen lag-length criterion), see the right panel of Table $9 .{ }^{17}$ We then compare the results to that of the $\tilde{\chi}_{\mathcal{I}}^{2}\left(\lambda_{\max }, t_{\gamma}^{\mathrm{ADF}}, t_{\gamma}^{\mathrm{ECR}}, \hat{F}\right)$ test.

Table 9 thus reports the frequencies for all possible pairs of outcomes. ${ }^{18}$ We see that when all tests reject $\mathcal{H}_{0}$, the meta test does so to. However, such cases of agreeing tests make up only $65 \%$

\footnotetext{
${ }^{17}$ For this purpose, we categorize the studies according to whether they use a residual- (i.e. those by Engle and Granger, 1987, or Phillips and Ouliaris, 1990) or system-based Johansen (1988) test. That is, we identify all Johansen tests with $\lambda_{\max }$ and all residual-based tests with $t_{\gamma}^{\mathrm{ADF}}$. Given the highly positive correlation within classes of tests (Gregory et al., 2004), this approximation is accurate. In five (58-53) cases of conflicting test results, the original studies do not report a cointegration test, being concerned with e.g. estimating cointegration vectors.

${ }^{18}$ Appendix E reports results for $\tilde{\chi}_{\mathcal{I}}^{2}\left(\lambda_{\max }, t_{\gamma}^{\mathrm{ADF}}\right)$; results for other (bootstrap) combination tests are available.
} 
Table 8: Rejection rates when combining $|\mathcal{I}|>2$ tests

\begin{tabular}{lllllll}
\hline \hline \multirow{2}{*}{ DGP } & $T$ & \multicolumn{2}{c}{ Size } & & \multicolumn{2}{c}{ Power } \\
\cline { 3 - 4 } \cline { 6 - 7 }$(\mathrm{A})$ & 50 & $\tilde{\chi}_{\mathcal{I}}^{2}(2)$ & $\tilde{\chi}_{\mathcal{I}}^{2}(4)$ & & $\tilde{\chi}_{\mathcal{I}}^{2}(2)$ & $\tilde{\chi}_{\mathcal{I}}^{2}(4)$ \\
& 75 & 0.061 & 0.071 & & 0.359 & 0.490 \\
& 100 & 0.055 & 0.068 & & 0.343 & 0.464 \\
& 150 & 0.054 & 0.056 & & 0.307 & 0.440 \\
& 200 & 0.044 & 0.047 & & 0.284 & 0.391 \\
$(\mathrm{~B})$ & 50 & 0.063 & 0.069 & & 0.100 & 0.114 \\
& 75 & 0.060 & 0.063 & & 0.157 & 0.171 \\
& 100 & 0.060 & 0.060 & & 0.269 & 0.267 \\
& 150 & 0.057 & 0.055 & & 0.591 & 0.531 \\
& 200 & 0.063 & 0.062 & & 0.880 & 0.810 \\
$(\mathrm{C})$ & 50 & 0.060 & 0.069 & & 0.310 & 0.330 \\
& 75 & 0.055 & 0.061 & & 0.285 & 0.309 \\
& 100 & 0.054 & 0.060 & & 0.268 & 0.281 \\
& 150 & 0.049 & 0.059 & & 0.258 & 0.271 \\
& 200 & 0.044 & 0.052 & & 0.239 & 0.255 \\
\hline
\end{tabular}

Average rejection rates at nominal level of $5 \%$. 5,000 replications. $U R_{\psi_{\mathcal{I}}}(|\mathcal{I}|)$ and $\tilde{\chi}_{\mathcal{I}}^{2}(|\mathcal{I}|)$ combine the $|\mathcal{I}|$ tests described in the text. For $\operatorname{DGP}(\mathrm{A})$, results are based on $R^{2}=0.25$.

$(=(52+51) / 159)$ of all data sets (tests). For the remaining $36 \%$ of data sets we have conflicting single tests and here our test turns out to be most useful. It allows the researcher to arrive at a definite conclusion. We find in $47 \%(=27 / 58)$ of the conflicting cases that the meta test does not reject $\mathcal{H}_{0}$. In the remaining $53 \%$ of the conflicting cases, however, the $\tilde{\chi}_{\mathcal{I}}^{2}$ test leads to a rejection of $\mathcal{H}_{0}$. Moreover, we note the following.

First, rejecting whenever at least one (but not all) of the tests rejected would have lead to a substantial overstatement of cointegration ( 58 vs. 31 cases according to the $\tilde{\chi}_{\mathcal{I}}^{2}$ test). Similarly, not rejecting whenever one test did not reject would have lead to an understatement of cointegration.

Second, the tests that have been 'preferred' in the actual studies tend to be more rejective than our meta test (37 vs. 29 rejections in 53 tests). This suggests that the evidence in favor of cointegration would have been somewhat less pronounced if the studies could have relied on a suitable meta test for cointegration. (Note that the preferred test being more rejective than the meta test here does not contradict the favorable power properties of the meta test found in Section 6 , as the latter can, and should, of course only be shown to be powerful in a class of level- $\alpha$ tests. Whether or not the way researchers identify their 'preferred' test leads to a level- $\alpha$ test or suffers from data-mining is impossible to say without knowledge of the decision process.)

Third, whether or not the preferred test rejected $\mathcal{H}_{0}$ does not seem to be informative on whether or not $\tilde{\chi}_{\mathcal{I}}^{2}$ rejects conditional on observing conflicting test results. This is reflected by very similar conditional probabilities: $27 / 58 \simeq 17 / 37 \simeq 7 / 16 \approx 0.45$. In other words, we cannot conclude from a published test result what the $\tilde{\chi}_{\mathcal{I}}^{2}$ test would indicate, conditional on the fact that a further single test leads to a conflicting test result. 
Table 9: Test results in applied studies and the $\tilde{\chi}_{\mathcal{I}}^{2}$ test

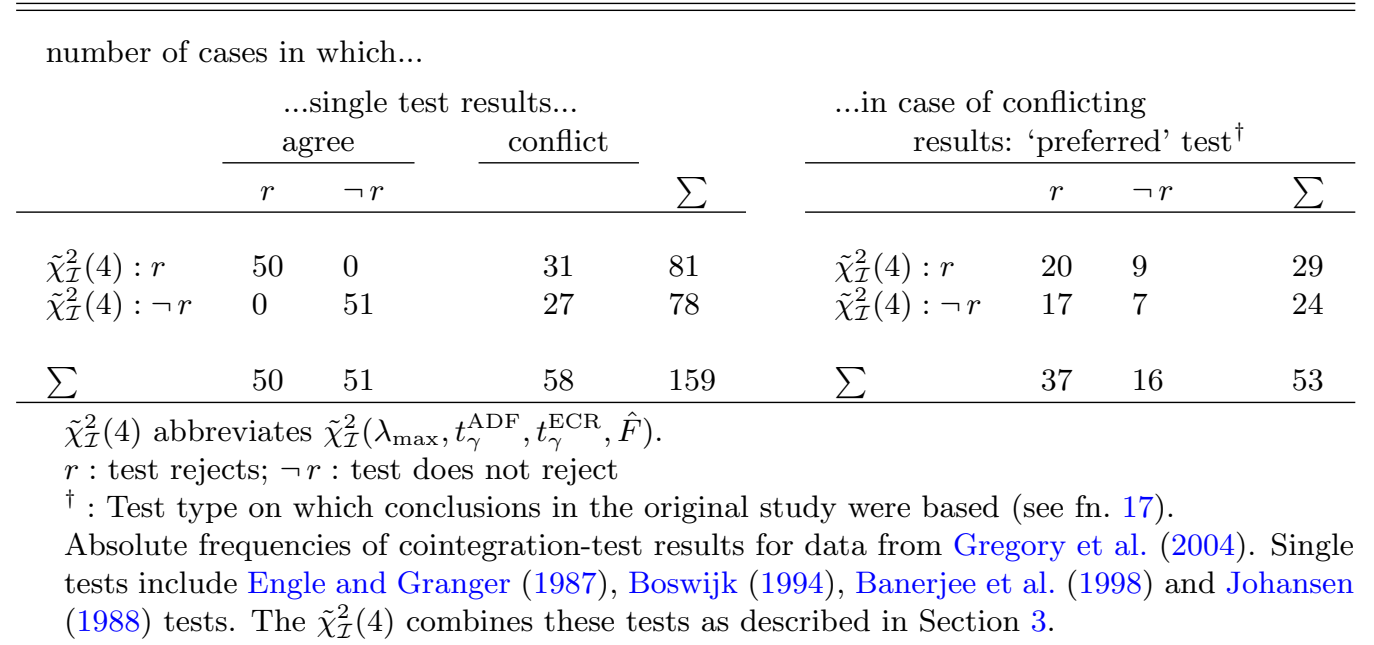

\section{Conclusion}

This paper proposes meta tests that combine information from different underlying tests for cointegration. The tests take into account the multiple testing nature of running more than one underlying test and hence control size. The meta tests are constructed by deriving the distribution of suitable aggregators of the underlying tests (e.g., Fisher's), by appropriately modifying the critical values of the underlying tests, as well as by using corresponding bootstrap methods. By contrast, running more than one test and then simply inferring about the hypothesis from the most rejective test leads to a significantly oversized test, as we have shown. Asymptotic and Monte Carlo results demonstrate the effectiveness of the proposed meta tests, establishing attractive power properties. An application of our test to a set of cointegration studies confirms its practical value. It yields an unambiguous test decision in cases of conflicting single test results.

The setup we put forward is fairly general and hence can be adopted to other testing problems for which several (imperfectly correlated) tests have been developed. Examples include testing for unit roots or heteroscedasticity. Essentially, what is needed is either the distribution of some suitable aggregator or a bootstrap method suitable for the phenomenon of interest. For the above mentioned testing problems such bootstrap methods would be the sieve and the wild bootstrap.

In practice, a major advantage of our proposed tests should be that they relieve the applied researcher from the discretionary and sometimes arbitrary choice of the cointegration test(s) she wants to rely on to reach a test decision.

\section{References}

Banerjee, A., Dolado, J. J., and Mestre, R. (1998), "Error-correction mechanism tests for cointegration in a singleequation framework," Journal of Time Series Analysis, 19(3), 267-283. 
Boswijk, H. P. (1994), "Testing for an unstable root in conditional and unconditional error correction models," Journal of Econometrics, 63, 37-60.

Breitung, J. (2001), "Rank tests for nonlinear cointegration," Journal of Business 8 Economic Statistics, 19(3), 331340 .

Demetrescu, M., Hassler, U., and Tarcolea, A.-I. (2006), "Combining significance of correlated statistics with application to panel data," Oxford Bulletin of Economics and Statistics, 68(5), 647-663.

Elliott, G., Jansson, M., and Pesavento, E. (2005), "Optimal power for testing potential cointegrating vectors with known parameters for nonstationarity," Journal of Business 83 Economic Statistics, 23(1), 34-48.

Engle, R. F., and Granger, C. W. J. (1987), "Co-integration and error correction: Representation, estimation, and testing," Econometrica, 55(2), 251-76.

Fisher, R. A. (1932) Statistical Methods for Research Workers, London: Oliver and Boyd.

Gregory, A. W., Haug, A. A., and Lomuto, N. (2004), "Mixed signals among tests for cointegration," Journal of Applied Econometrics, 19(1), 89-98.

Hamilton, J. D. (1994) Time Series Analysis, Princeton: Princeton University Press.

Hartung, J. (1999), "A note on combining dependent tests of significance," Biometrical Journal, 41, 849-855.

Harvey, D. I., Leybourne, S. J., and Taylor, A. M. R. (2009), "Unit root testing in practice: Dealing with uncertainty over the trend and initial condition," Econometric Theory to appear.

Haug, A. A. (1996), "Tests for cointegration: A Monte Carlo comparison," Journal of Econometrics, 71, 89-115.

Horowitz, J. L. (2001), "The bootstrap," In Handbook of Econometrics, ed. James J. Heckman and Edward E. Leamer, vol. 5, Amsterdam: Elsevier chapter 52, pp. 3159-3228.

Johansen, S. (1988), "Statistical analysis of cointegration vectors," Journal of Economic Dynamics and Control, $12(2-3), 231-254$.

_ (1995) Likelihood-Based Inference in Cointegrated Vector Autoregressive Models, Oxford University Press.

Kremers, J. J., Ericsson, N. R., and Dolado, J. J. (1992), "The power of cointegration tests," Oxford Bulletin of Economics and Statistics, 54(3), 325-348.

Lütkepohl, H. (2005) New Introduction to Multiple Time Series Analysis, Berlin: Springer.

MacKinnon, J. G. (1996), "Numerical distribution functions for unit root and cointegration tests," Journal of Applied Econometrics, 11(6), 601-618.

Palm, F. C., Smeekes, S., and Urbain, J.-P. (2009), "A sieve bootstrap test for cointegration in a conditional error correction model," Econometric Theory to appear.

Paparoditis, E., and Politis, D. N. (2003), "Residual-based block bootstrap for unit root testing," Econometrica, $71(3), 813-855$.

Pesavento, E. (2004), "Analytical evaluation of the power of tests for the absence of cointegration," Journal of Econometrics, 122, 349-384.

- (2007), "Residuals-based tests for the null of no-cointegration: An analytical comparison," Journal of Time Series Analysis, 28(1), 111-137.

Phillips, P. C. B., and Ouliaris, S. (1990), "Asymptotic properties of residual based tests for cointegration," Econometrica, 58(1), 165-193.

Swensen, A. R. (2006), "Bootstrap algorithms for testing and determining the cointegration rank in VAR models," Econometrica, 74(6), 1699-1714. 


\section{Appendix A Further critical values and correction factors}

Table A.1: Critical values for the $\tilde{\chi}_{\mathcal{I}}^{2}$ test

\begin{tabular}{|c|c|c|c|c|c|c|c|c|c|c|c|c|}
\hline \multirow[b]{2}{*}{$K-1$} & \multicolumn{12}{|c|}{ case } \\
\hline & (i) & (ii) & (iii) & (i) & (ii) & (iii) & (i) & (ii) & (iii) & (i) & (ii) & (iii) \\
\hline \multicolumn{13}{|c|}{$\alpha=0.01$} \\
\hline & \multicolumn{3}{|c|}{$t_{\gamma}^{\mathrm{ADF}}$ and $\lambda_{\max }$} & \multicolumn{3}{|c|}{$\hat{F}$ and $\lambda_{\max }$} & \multicolumn{3}{|c|}{$\hat{F}$ and $t_{\gamma}^{\mathrm{ECR}}$} & \multicolumn{3}{|c|}{$\hat{F}$ and $t_{\gamma}^{\mathrm{ADF}}$} \\
\hline 1 & 16.948 & 17.304 & 17.289 & 17.077 & 17.175 & 17.066 & 17.827 & 18.201 & 18.230 & 16.551 & 17.390 & 17.572 \\
\hline 2 & 16.651 & 16.679 & 16.720 & 16.443 & 16.355 & 16.227 & 17.888 & 18.051 & 18.176 & 16.361 & 16.686 & 17.078 \\
\hline 3 & 16.236 & 16.259 & 16.263 & 15.787 & 15.814 & 15.777 & 17.831 & 17.951 & 18.069 & 16.137 & 16.430 & 16.795 \\
\hline 4 & 15.871 & 15.845 & 15.973 & 15.384 & 15.497 & 15.430 & 17.763 & 17.912 & 18.017 & 16.074 & 16.396 & 16.493 \\
\hline 5 & 15.626 & 15.701 & 15.666 & 15.241 & 15.143 & 15.202 & 17.889 & 17.813 & 17.937 & 16.011 & 16.201 & 16.295 \\
\hline 6 & 15.412 & 15.348 & 15.467 & 15.015 & 15.038 & 14.995 & 17.773 & 17.710 & 17.937 & 15.858 & 15.997 & 16.326 \\
\hline & \multicolumn{3}{|c|}{$\hat{F}, \lambda_{\max }$ and $t_{\gamma}^{\mathrm{ADF}}$} & \multicolumn{3}{|c|}{$\hat{F}, \lambda_{\max }$ and $t_{\gamma}^{\mathrm{ECR}}$} & \multicolumn{3}{|c|}{$\hat{F}, \lambda_{\max }, t_{\gamma}^{\mathrm{ADF}}, t_{\gamma}^{\mathrm{ECR}}$} & & & \\
\hline 1 & 24.174 & 25.263 & 25.420 & 25.151 & 25.718 & 25.726 & 32.713 & 33.969 & 34.334 & & & \\
\hline 2 & 23.595 & 23.855 & 24.091 & 24.369 & 24.501 & 24.623 & 31.793 & 32.077 & 32.601 & & & \\
\hline 3 & 22.685 & 23.026 & 23.446 & 23.485 & 23.731 & 23.936 & 30.651 & 31.169 & 31.742 & & & \\
\hline 4 & 22.256 & 22.498 & 22.681 & 23.144 & 23.344 & 23.461 & 30.088 & 30.774 & 30.836 & & & \\
\hline 5 & 21.924 & 22.020 & 22.058 & 22.799 & 22.974 & 23.003 & 29.800 & 29.850 & 30.113 & & & \\
\hline 6 & 21.686 & 21.729 & 21.887 & 22.633 & 22.548 & 22.677 & 29.222 & 29.544 & 29.962 & & & \\
\hline \multicolumn{13}{|c|}{$\alpha=0.1$} \\
\hline & \multicolumn{3}{|c|}{$t_{\gamma}^{\mathrm{ADF}}$ and $\lambda_{\max }$} & \multicolumn{3}{|c|}{$\hat{F}$ and $\lambda_{\max }$} & \multicolumn{3}{|c|}{$\hat{F}$ and $t_{\gamma}^{\mathrm{ECR}}$} & \multicolumn{3}{|c|}{$\hat{F}$ and $t_{\gamma}^{\mathrm{ADF}}$} \\
\hline 1 & 8.612 & 8.678 & 8.686 & 8.614 & 8.596 & 8.588 & 8.895 & 9.085 & 9.120 & 8.478 & 8.739 & 8.892 \\
\hline 2 & 8.457 & 8.479 & 8.451 & 8.368 & 8.390 & 8.351 & 8.907 & 9.031 & 9.062 & 8.434 & 8.607 & 8.702 \\
\hline 3 & 8.350 & 8.363 & 8.352 & 8.251 & 8.241 & 8.254 & 8.868 & 8.980 & 9.049 & 8.370 & 8.494 & 8.611 \\
\hline 4 & 8.290 & 8.301 & 8.272 & 8.199 & 8.151 & 8.167 & 8.915 & 8.957 & 9.015 & 8.346 & 8.478 & 8.555 \\
\hline 5 & 8.221 & 8.242 & 8.276 & 8.150 & 8.105 & 8.127 & 8.887 & 8.939 & 9.009 & 8.353 & 8.440 & 8.563 \\
\hline \multirow[t]{2}{*}{6} & 8.165 & 8.200 & 8.199 & 8.094 & 8.093 & 8.076 & 8.892 & 8.899 & 8.973 & 8.366 & 8.456 & 8.507 \\
\hline & \multicolumn{3}{|c|}{$\hat{F}, \lambda_{\max }$ and $t_{\gamma}^{\mathrm{ADF}}$} & \multicolumn{3}{|c|}{$\hat{F}, \lambda_{\max }$ and $t_{\gamma}^{\mathrm{ECR}}$} & \multicolumn{3}{|c|}{$\hat{F}, \lambda_{\max }, t_{\gamma}^{\mathrm{ADF}}, t_{\gamma}^{\mathrm{ECR}}$} & & & \\
\hline 1 & 12.570 & 12.761 & 12.855 & 12.542 & 12.748 & 12.863 & 16.593 & 16.964 & 17.187 & & & \\
\hline 2 & 12.218 & 12.378 & 12.374 & 12.265 & 12.379 & 12.358 & 16.171 & 16.444 & 16.507 & & & \\
\hline 3 & 12.008 & 12.075 & 12.177 & 12.031 & 12.175 & 12.244 & 15.920 & 16.097 & 16.239 & & & \\
\hline 4 & 11.873 & 11.962 & 12.008 & 12.007 & 12.059 & 12.108 & 15.776 & 15.938 & 16.086 & & & \\
\hline 5 & 11.807 & 11.857 & 11.915 & 11.971 & 11.999 & 12.044 & 15.681 & 15.804 & 15.989 & & & \\
\hline 6 & 11.711 & 11.773 & 11.826 & 11.880 & 11.970 & 11.995 & 15.644 & 15.746 & 15.872 & & & \\
\hline
\end{tabular}

$1 \%$ - and $10 \%$-critical values for combination tests based on $\tilde{\chi}_{\mathcal{I}}^{2} \cdot t_{\gamma}^{\mathrm{ADF}}$ is from Engle and Granger (1987), $\lambda_{\max }$ from Johansen (1988), $\hat{F}$ from Boswijk (1994) and $t_{\gamma}^{\mathrm{ECR}}$ from Banerjee et al. (1998). 
Table A.2: Correction Factors for the minimum $p$-value test.

\begin{tabular}{|c|c|c|c|c|c|c|}
\hline \multirow[b]{2}{*}{$K-1$} & \multicolumn{6}{|c|}{ case } \\
\hline & (i) & (ii) & (iii) & (i) & (ii) & (iii) \\
\hline \multicolumn{7}{|c|}{$\alpha=0.01$} \\
\hline & \multicolumn{3}{|c|}{$t_{\gamma}^{\mathrm{ADF}}$ and $\lambda_{\max }$} & \multicolumn{3}{|c|}{$\hat{F}$ and $t_{\gamma}^{\mathrm{ECR}}$} \\
\hline 1 & 0.006 & 0.006 & 0.006 & 0.007 & 0.008 & 0.008 \\
\hline 2 & 0.006 & 0.006 & 0.006 & 0.007 & 0.008 & 0.008 \\
\hline 3 & 0.006 & 0.006 & 0.006 & 0.007 & 0.007 & 0.008 \\
\hline 4 & 0.005 & 0.005 & 0.005 & 0.007 & 0.007 & 0.007 \\
\hline 5 & 0.005 & 0.005 & 0.005 & 0.007 & 0.007 & 0.007 \\
\hline 6 & 0.005 & 0.005 & 0.005 & 0.007 & 0.007 & 0.007 \\
\hline \multicolumn{7}{|c|}{$\alpha=0.05$} \\
\hline & \multicolumn{3}{|c|}{$t_{\gamma}^{\mathrm{ADF}}$ and $\lambda_{\max }$} & \multicolumn{3}{|c|}{$\hat{F}$ and $t_{\gamma}^{\mathrm{ECR}}$} \\
\hline 1 & 0.031 & 0.033 & 0.033 & 0.038 & 0.041 & 0.043 \\
\hline 2 & 0.030 & 0.030 & 0.030 & 0.037 & 0.038 & 0.040 \\
\hline 3 & 0.029 & 0.029 & 0.029 & 0.036 & 0.038 & 0.039 \\
\hline 4 & 0.028 & 0.028 & 0.028 & 0.036 & 0.037 & 0.038 \\
\hline 5 & 0.028 & 0.028 & 0.028 & 0.035 & 0.036 & 0.037 \\
\hline 6 & 0.027 & 0.027 & 0.028 & 0.035 & 0.035 & 0.037 \\
\hline \multicolumn{7}{|c|}{$\alpha=0.1$} \\
\hline & \multicolumn{3}{|c|}{$t_{\gamma}^{\mathrm{ADF}}$ and $\lambda_{\max }$} & \multicolumn{3}{|c|}{$\hat{F}$ and $t_{\gamma}^{\mathrm{ECR}}$} \\
\hline 1 & 0.064 & 0.067 & 0.067 & 0.077 & 0.083 & 0.086 \\
\hline 2 & 0.061 & 0.062 & 0.062 & 0.075 & 0.079 & 0.081 \\
\hline 3 & 0.059 & 0.059 & 0.060 & 0.074 & 0.076 & 0.079 \\
\hline 4 & 0.058 & 0.058 & 0.058 & 0.072 & 0.075 & 0.077 \\
\hline 5 & 0.057 & 0.057 & 0.057 & 0.072 & 0.074 & 0.075 \\
\hline 6 & 0.056 & 0.056 & 0.056 & 0.071 & 0.073 & 0.075 \\
\hline
\end{tabular}

Correction Factors for the minimum $p$-value test. 


\section{Appendix B Local Asymptotic Power, further results}

Table B.1: Local Asymptotic Power

\begin{tabular}{|c|c|c|c|c|c|}
\hline$-c$ & 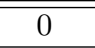 & 5 & 10 & 15 & 20 \\
\hline \multicolumn{6}{|l|}{$R^{2}=0$} \\
\hline$\tilde{\chi}_{\mathcal{I}}^{2}\left(\hat{F}, t_{\gamma}^{\mathrm{ECR}}\right)$ & 0.050 & 0.153 & 0.404 & 0.716 & 0.917 \\
\hline$\tilde{\chi}_{\mathcal{I}}^{2}\left(t_{\gamma}^{\mathrm{ADF}}, \lambda_{\max }\right)$ & 0.050 & 0.120 & 0.311 & 0.595 & 0.841 \\
\hline$\tilde{\chi}_{\mathcal{I}}^{2}\left(\hat{F}, t_{\gamma}^{\mathrm{ECR}}, t_{\gamma}^{\mathrm{ADF}}, \lambda_{\max }\right)$ & 0.050 & 0.153 & 0.403 & 0.709 & 0.913 \\
\hline$U R_{\psi_{\mathcal{I}}}\left(\hat{F}, t_{\gamma}^{\mathrm{ECR}}\right)$ & 0.049 & 0.137 & 0.372 & 0.682 & 0.898 \\
\hline$U R_{\psi_{\mathcal{I}}}\left(t_{\gamma}^{\mathrm{ADF}}, \lambda_{\max }\right)$ & 0.050 & 0.103 & 0.280 & 0.555 & 0.813 \\
\hline$\hat{F}$ & 0.050 & 0.114 & 0.319 & 0.616 & 0.861 \\
\hline$t_{\gamma}^{\mathrm{ECR}}$ & 0.050 & 0.175 & 0.450 & 0.762 & 0.939 \\
\hline$\lambda_{\max }$ & 0.050 & 0.076 & 0.187 & 0.391 & 0.641 \\
\hline$t_{\gamma}^{\mathrm{ADF}}$ & 0.050 & 0.134 & 0.364 & 0.669 & 0.892 \\
\hline \multicolumn{6}{|l|}{$R^{2}=0.25$} \\
\hline$\tilde{\chi}_{\mathcal{I}}^{2}\left(\hat{F}, t_{\gamma}^{\mathrm{ECR}}\right)$ & 0.049 & 0.196 & 0.561 & 0.862 & 0.974 \\
\hline$\tilde{\chi}_{\mathcal{I}}^{2}\left(t_{\gamma}^{\mathrm{ADF}}, \lambda_{\max }\right)$ & 0.049 & 0.126 & 0.377 & 0.714 & 0.933 \\
\hline$\tilde{\chi}_{\mathcal{I}}^{2}\left(\hat{F}, t_{\gamma}^{\mathrm{ECR}}, t_{\gamma}^{\mathrm{ADF}}, \lambda_{\max }\right)$ & 0.050 & 0.179 & 0.523 & 0.847 & 0.975 \\
\hline$U R_{\psi_{\mathcal{I}}}\left(\hat{F}, t_{\gamma}^{\mathrm{ECR}}\right)$ & 0.049 & 0.172 & 0.511 & 0.827 & 0.965 \\
\hline$U R_{\psi_{\mathcal{I}}}\left(t_{\gamma}^{\mathrm{ADF}}, \lambda_{\max }\right)$ & 0.046 & 0.116 & 0.337 & 0.647 & 0.891 \\
\hline$\hat{F}$ & 0.049 & 0.174 & 0.513 & 0.819 & 0.958 \\
\hline$t_{\gamma}^{\mathrm{ECR}}$ & 0.050 & 0.198 & 0.558 & 0.864 & 0.976 \\
\hline$\lambda_{\max }$ & 0.047 & 0.105 & 0.312 & 0.614 & 0.867 \\
\hline$t_{\gamma}^{\mathrm{ADF}}$ & 0.048 & 0.120 & 0.331 & 0.625 & 0.871 \\
\hline \multicolumn{6}{|l|}{$R^{2}=0.5$} \\
\hline$\tilde{\chi}_{\mathcal{I}}^{2}\left(\hat{F}, t_{\gamma}^{\mathrm{ECR}}\right)$ & 0.050 & 0.293 & 0.757 & 0.954 & 0.995 \\
\hline$\tilde{\chi}_{\mathcal{I}}^{2}\left(t_{\gamma}^{\mathrm{ADF}}, \lambda_{\max }\right)$ & 0.053 & 0.157 & 0.541 & 0.893 & 0.991 \\
\hline$\tilde{\chi}_{\mathcal{I}}^{2}\left(\hat{F}, t_{\gamma}^{\mathrm{ECR}}, t_{\gamma}^{\mathrm{ADF}}, \lambda_{\max }\right)$ & 0.053 & 0.254 & 0.723 & 0.958 & 0.997 \\
\hline$U R_{\psi_{\mathcal{I}}}\left(\hat{F}, t_{\gamma}^{\mathrm{ECR}}\right)$ & 0.049 & 0.288 & 0.729 & 0.942 & 0.993 \\
\hline$U R_{\psi_{\mathcal{I}}}\left(t_{\gamma}^{\mathrm{ADF}}, \lambda_{\max }\right)$ & 0.051 & 0.172 & 0.532 & 0.861 & 0.982 \\
\hline$\hat{F}$ & 0.052 & 0.328 & 0.763 & 0.949 & 0.994 \\
\hline$t_{\gamma}^{\mathrm{ECR}}$ & 0.050 & 0.230 & 0.689 & 0.938 & 0.993 \\
\hline$\lambda_{\max }$ & 0.049 & 0.192 & 0.578 & 0.888 & 0.988 \\
\hline$t_{\gamma}^{\mathrm{ADF}}$ & 0.054 & 0.106 & 0.284 & 0.581 & 0.842 \\
\hline \multicolumn{6}{|l|}{$R^{2}=0.75$} \\
\hline$\tilde{\chi}_{\mathcal{I}}^{2}\left(\hat{F}, t_{\gamma}^{\mathrm{ECR}}\right)$ & 0.052 & 0.573 & 0.954 & 0.997 & 1.000 \\
\hline$\tilde{\chi}_{\mathcal{I}}^{2}\left(t_{\gamma}^{\mathrm{ADF}}, \lambda_{\max }\right)$ & 0.051 & 0.344 & 0.898 & 0.997 & 1.000 \\
\hline$\tilde{\chi}_{\mathcal{I}}^{2}\left(\hat{F}, t_{\gamma}^{\mathrm{ECR}}, t_{\gamma}^{\mathrm{ADF}}, \lambda_{\max }\right)$ & 0.051 & 0.516 & 0.955 & 0.999 & 1.000 \\
\hline$U R_{\psi_{\mathcal{I}}}\left(\hat{F}, t_{\gamma}^{\mathrm{ECR}}\right)$ & 0.052 & 0.616 & 0.953 & 0.997 & 1.000 \\
\hline$U R_{\psi_{\mathcal{I}}}\left(t_{\gamma}^{\mathrm{ADF}}, \lambda_{\max }\right)$ & 0.050 & 0.431 & 0.914 & 0.997 & 1.000 \\
\hline$\hat{F}$ & 0.052 & 0.659 & 0.963 & 0.997 & 1.000 \\
\hline$t_{\gamma}^{\mathrm{ECR}}$ & 0.050 & 0.369 & 0.892 & 0.992 & 1.000 \\
\hline$\lambda_{\max }$ & 0.050 & 0.495 & 0.942 & 0.998 & 1.000 \\
\hline$t_{\gamma}^{\mathrm{ADF}}$ & 0.051 & 0.079 & 0.235 & 0.523 & 0.805 \\
\hline
\end{tabular}

Case $(i)$. See notes to Table 3 . 
Table B.2: Local Asymptotic Power

\begin{tabular}{|c|c|c|c|c|c|}
\hline$-c$ & 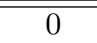 & 5 & 10 & 15 & 20 \\
\hline \multicolumn{6}{|l|}{$R^{2}=0$} \\
\hline$\tilde{\chi}_{\mathcal{I}}^{2}\left(\hat{F}, t_{\gamma}^{\mathrm{ECR}}\right)$ & 0.050 & 0.073 & 0.148 & 0.290 & 0.487 \\
\hline$\tilde{\chi}_{\mathcal{I}}^{2}\left(t_{\gamma}^{\mathrm{ADF}}, \lambda_{\max }\right)$ & 0.050 & 0.069 & 0.132 & 0.253 & 0.423 \\
\hline$\tilde{\chi}_{\mathcal{I}}^{2}\left(\hat{F}, t_{\gamma}^{\mathrm{ECR}}, t_{\gamma}^{\mathrm{ADF}}, \lambda_{\max }\right)$ & 0.050 & 0.074 & 0.151 & 0.294 & 0.490 \\
\hline$U R_{\psi_{\mathcal{I}}}\left(\hat{F}, t_{\gamma}^{\mathrm{ECR}}\right)$ & 0.049 & 0.070 & 0.142 & 0.279 & 0.471 \\
\hline$U R_{\psi_{\mathcal{I}}}\left(t_{\gamma}^{\mathrm{ADF}}, \lambda_{\max }\right)$ & 0.051 & 0.064 & 0.116 & 0.230 & 0.392 \\
\hline$\hat{F}$ & 0.050 & 0.070 & 0.138 & 0.271 & 0.457 \\
\hline$t_{\gamma}^{\mathrm{ECR}}$ & 0.050 & 0.076 & 0.155 & 0.305 & 0.508 \\
\hline$\lambda_{\max }$ & 0.050 & 0.054 & 0.092 & 0.165 & 0.283 \\
\hline$t_{\gamma}^{\mathrm{ADF}}$ & 0.050 & 0.074 & 0.150 & 0.290 & 0.486 \\
\hline \multicolumn{6}{|l|}{$R^{2}=0.25$} \\
\hline$\tilde{\chi}_{\mathcal{I}}^{2}\left(\hat{F}, t_{\gamma}^{\mathrm{ECR}}\right)$ & 0.048 & 0.081 & 0.191 & 0.405 & 0.668 \\
\hline$\tilde{\chi}_{\mathcal{I}}^{2}\left(t_{\gamma}^{\mathrm{ADF}}, \lambda_{\max }\right)$ & 0.050 & 0.072 & 0.127 & 0.267 & 0.495 \\
\hline$\tilde{\chi}_{\mathcal{I}}^{2}\left(\hat{F}, t_{\gamma}^{\mathrm{ECR}}, t_{\gamma}^{\mathrm{ADF}}, \lambda_{\max }\right)$ & 0.049 & 0.084 & 0.194 & 0.406 & 0.664 \\
\hline$U R_{\psi_{\mathcal{I}}}\left(\hat{F}, t_{\gamma}^{\mathrm{ECR}}\right)$ & 0.051 & 0.069 & 0.121 & 0.247 & 0.456 \\
\hline$U R_{\psi_{\mathcal{I}}}\left(t_{\gamma}^{\mathrm{ADF}}, \lambda_{\max }\right)$ & 0.050 & 0.079 & 0.171 & 0.364 & 0.626 \\
\hline$\hat{F}$ & 0.047 & 0.083 & 0.199 & 0.412 & 0.668 \\
\hline$t_{\gamma}^{\mathrm{ECR}}$ & 0.050 & 0.083 & 0.183 & 0.388 & 0.652 \\
\hline$\lambda_{\max }$ & 0.050 & 0.067 & 0.123 & 0.261 & 0.471 \\
\hline$t_{\gamma}^{\mathrm{ADF}}$ & 0.050 & 0.070 & 0.115 & 0.222 & 0.398 \\
\hline \multicolumn{6}{|l|}{$R^{2}=0.5$} \\
\hline$\tilde{\chi}_{\mathcal{I}}^{2}\left(\hat{F}, t_{\gamma}^{\mathrm{ECR}}\right)$ & 0.049 & 0.089 & 0.285 & 0.621 & 0.874 \\
\hline$\tilde{\chi}_{\mathcal{I}}^{2}\left(t_{\gamma}^{\mathrm{ADF}}, \lambda_{\max }\right)$ & 0.050 & 0.063 & 0.146 & 0.386 & 0.699 \\
\hline$\tilde{\chi}_{\mathcal{I}}^{2}\left(\hat{F}, t_{\gamma}^{\mathrm{ECR}}, t_{\gamma}^{\mathrm{ADF}}, \lambda_{\max }\right)$ & 0.049 & 0.080 & 0.231 & 0.552 & 0.840 \\
\hline$U R_{\psi_{\mathcal{I}}}\left(\hat{F}, t_{\gamma}^{\mathrm{ECR}}\right)$ & 0.049 & 0.102 & 0.318 & 0.648 & 0.882 \\
\hline$U R_{\psi_{\mathcal{I}}}\left(t_{\gamma}^{\mathrm{ADF}}, \lambda_{\max }\right)$ & 0.049 & 0.069 & 0.179 & 0.439 & 0.734 \\
\hline$\hat{F}$ & 0.048 & 0.108 & 0.339 & 0.669 & 0.891 \\
\hline$t_{\gamma}^{\mathrm{ECR}}$ & 0.050 & 0.079 & 0.228 & 0.537 & 0.823 \\
\hline$\lambda_{\max }$ & 0.048 & 0.078 & 0.221 & 0.511 & 0.794 \\
\hline$t_{\gamma}^{\mathrm{ADF}}$ & 0.050 & 0.052 & 0.077 & 0.151 & 0.292 \\
\hline \multicolumn{6}{|l|}{$R^{2}=0.75$} \\
\hline$\tilde{\chi}_{\mathcal{I}}^{2}\left(\hat{F}, t_{\gamma}^{\mathrm{ECR}}\right)$ & 0.051 & 0.134 & 0.596 & 0.923 & 0.993 \\
\hline$\tilde{\chi}_{\mathcal{I}}^{2}\left(t_{\gamma}^{\mathrm{ADF}}, \lambda_{\max }\right)$ & 0.054 & 0.069 & 0.356 & 0.811 & 0.983 \\
\hline$\tilde{\chi}_{\mathcal{I}}^{2}\left(\hat{F}, t_{\gamma}^{\mathrm{ECR}}, t_{\gamma}^{\mathrm{ADF}}, \lambda_{\max }\right)$ & 0.053 & 0.107 & 0.524 & 0.906 & 0.993 \\
\hline$U R_{\psi_{\mathcal{I}}}\left(\hat{F}, t_{\gamma}^{\mathrm{ECR}}\right)$ & 0.050 & 0.196 & 0.689 & 0.946 & 0.995 \\
\hline$U R_{\psi_{\mathcal{I}}}\left(t_{\gamma}^{\mathrm{ADF}}, \lambda_{\max }\right)$ & 0.053 & 0.117 & 0.531 & 0.907 & 0.993 \\
\hline$\hat{F}$ & 0.052 & 0.216 & 0.714 & 0.952 & 0.996 \\
\hline$t_{\gamma}^{\mathrm{ECR}}$ & 0.050 & 0.077 & 0.385 & 0.801 & 0.970 \\
\hline$\lambda_{\max }$ & 0.051 & 0.153 & 0.607 & 0.937 & 0.996 \\
\hline$t_{\gamma}^{\mathrm{ADF}}$ & 0.054 & 0.029 & 0.035 & 0.071 & 0.166 \\
\hline
\end{tabular}

Case (iii). See notes to Table B.1. 
Figure B.1: Local asymptotic power as a function of $R^{2}, c=-5$
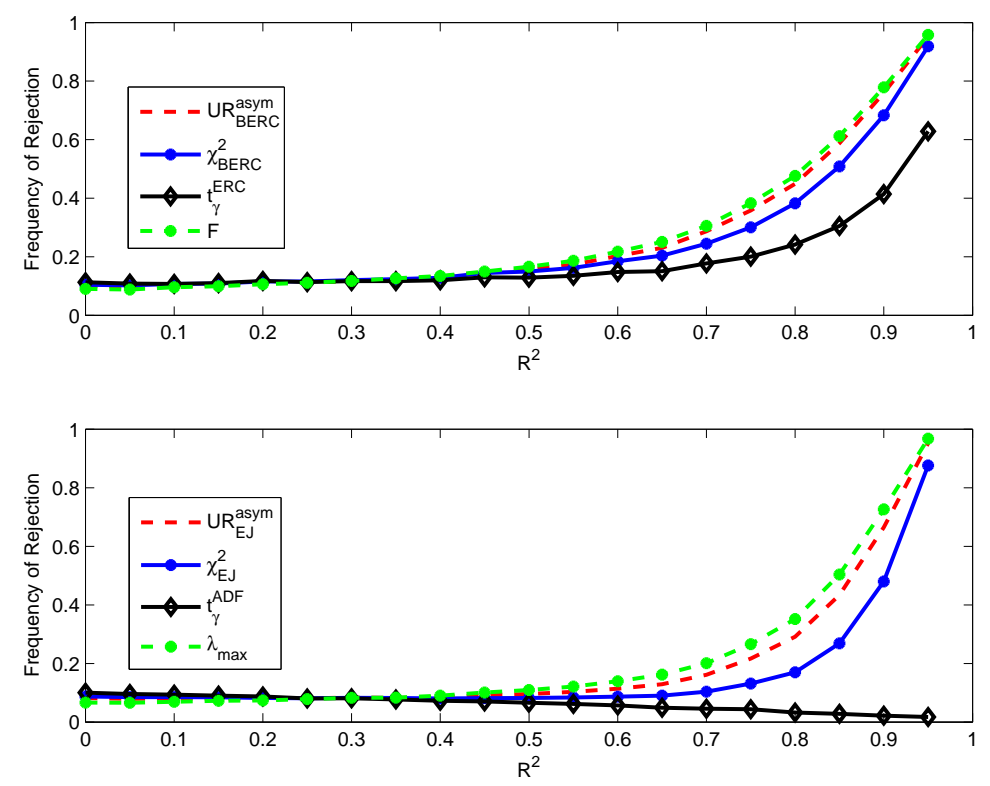

Results are for the demeaned case (ii). $\chi_{\text {BERC }}^{2}$ is our Fisher test (6) based on Boswijk's and Banerjee et al.'s tests. $\chi_{\mathrm{EJ}}^{2}$ is based on Engle and Granger's and Johansen's tests. $U R_{\mathrm{BERC}}^{\mathrm{asym}}$ and $U R_{\mathrm{EJ}}^{\text {asym }}$ are the corresponding asymmetric $U R_{\psi_{\mathcal{I}}}$ test (9). The single tests' power curves are for comparison.

\section{Appendix C Alternative Bootstrap Tests}

This Appendix describes an alternative bootstrap approach that makes somewhat stronger assumptions about the joint distribution of the $\xi_{i}$. Its power was slightly superior to the Fishertest version in our simulations (detailed results are available). Define a probit representation by $\Phi^{-1}\left(p_{i}\right)=: s_{i}$. Asymptotically, the $s_{i}$ are marginally standard normal under $\mathcal{H}_{0}$. Let $\boldsymbol{s}=$ $\left(s_{1}, \ldots, s_{|\mathcal{I}|}\right)^{\prime}$. Hartung (1999) additionally assumes joint normality for $\boldsymbol{s}$, denoted $\boldsymbol{s} \sim \mathcal{N}(\mathbf{0}, \boldsymbol{\Sigma})$. Under this assumption, we have $\boldsymbol{\iota}^{\prime} \boldsymbol{s} \sim \mathcal{N}\left(\mathbf{0}, \boldsymbol{\iota}^{\prime} \boldsymbol{\Sigma} \boldsymbol{\iota}\right)$, where $\boldsymbol{\iota}=(1, \ldots, 1)^{\prime}$. This leads to a standardized meta test statistic, $\tau=\boldsymbol{\iota}^{\prime} \boldsymbol{s} /\left(\boldsymbol{\iota}^{\prime} \boldsymbol{\Sigma} \boldsymbol{\iota}\right)^{1 / 2} . \tau$ is standard normal under $\mathcal{H}_{0}$ and joint normality. Fortunately, Demetrescu et al. (2006) show that this assumption is not necessary. As a practical matter, we rely on the following bootstrap method to provide a feasible estimator of $\boldsymbol{\Sigma}$.

\section{Algorithm 2.}

1. - 6. As in Algorithm 1.

7. Obtain the corresponding probit representation of each test statistic, $s_{i, b}^{*}=\Phi^{-1}\left(p_{i, b}^{*}\right)$, stacked in $\boldsymbol{s}_{b}^{*}=\left(s_{1, b}^{*}, \ldots, s_{|\mathcal{I}|, b}^{*}\right)^{\prime}$. Correspondingly, obtain $s_{i}^{*}=\Phi^{-1}\left(p_{i}^{*}\right)$ and $\boldsymbol{s}^{*}=\left(s_{1}^{*}, \ldots, s_{|\mathcal{I}|}^{*}\right)^{\prime}$.

8. Letting $\overline{\boldsymbol{s}}^{*}:=\frac{1}{B} \sum_{b} \boldsymbol{s}_{b}^{*}$, estimate the covariance matrix $\boldsymbol{\Sigma}$ of the probits of the tests by $\Sigma^{*}=\frac{1}{B} \sum_{b}\left(s_{b}^{*}-\bar{s}^{*}\right)\left(s_{b}^{*}-\bar{s}^{*}\right)^{\prime}$.

This Algorithm provides a feasible version of the test statistic $\tau, \tau^{*}=\frac{\boldsymbol{\iota}^{\prime} \boldsymbol{s}^{*}}{\sqrt{\boldsymbol{\iota}^{\prime} \boldsymbol{\Sigma}^{*} \boldsymbol{\iota}}}$. We reject $\mathcal{H}_{0}$ at level $\alpha$ if $\tau^{*}<\Phi^{-1}(\alpha)$. The following Lemma provides a useful consistency property of the test. 
Lemma 5. If (i) $\alpha<1 / 2$ and (ii) all $s_{i}$ reject at level $\alpha$, then $\tau^{*}$ rejects $\mathcal{H}_{0}$ at least at level $\alpha$.

Proof. Recall that $\Phi^{-1}(\alpha)<0$ for $\alpha<1 / 2$. Then, it follows from (ii) that $s_{i}<\Phi^{-1}(\alpha)<0$ for all $i=1, \ldots,|\mathcal{I}|$. Hence, $\iota^{\prime} s<0$. Further, since the entries of the positive semi-definite correlation matrix $\boldsymbol{\Sigma}^{*}$ are bounded by 1 and -1 , we have $\sqrt{\boldsymbol{\iota}^{\prime} \boldsymbol{\Sigma}^{*} \boldsymbol{\iota}} \leq|\mathcal{I}|$. Thus,

$$
\tau^{*}=\frac{\iota^{\prime} \boldsymbol{s}}{\sqrt{\boldsymbol{\iota}^{\prime} \Sigma^{*} \iota}} \leq \frac{\boldsymbol{\iota}^{\prime} \boldsymbol{s}}{|\mathcal{I}|}<\Phi^{-1}(\alpha)
$$

\section{Appendix D Additional Simulation Results}

Table D.1: Small-sample power, DGP(A), further $R^{2} \mathrm{~s}$

\begin{tabular}{|c|c|c|c|c|c|c|c|c|c|}
\hline DGP & $T$ & $\hat{\hat{F}}$ & $t_{\gamma}^{\mathrm{ECR}}$ & $\tilde{\chi}_{\mathcal{I}}^{2}$ & $U R_{\psi_{\mathcal{I}}}$ & $\lambda_{\max }$ & $t_{\gamma}^{\mathrm{ADF}}$ & $\tilde{\chi}_{\mathcal{I}}^{2}$ & $U R_{\psi_{\mathcal{I}}}$ \\
\hline \multirow[t]{5}{*}{$R^{2}=0$} & 50 & 0.401 & 0.427 & 0.418 & 0.411 & 0.194 & 0.440 & 0.349 & 0.380 \\
\hline & 75 & 0.370 & 0.407 & 0.395 & 0.387 & 0.192 & 0.406 & 0.323 & 0.341 \\
\hline & 100 & 0.343 & 0.376 & 0.364 & 0.356 & 0.177 & 0.369 & 0.300 & 0.315 \\
\hline & 150 & 0.319 & 0.353 & 0.341 & 0.329 & 0.178 & 0.335 & 0.284 & 0.294 \\
\hline & 200 & 0.301 & 0.331 & 0.322 & 0.311 & 0.173 & 0.320 & 0.263 & 0.275 \\
\hline \multirow[t]{5}{*}{$R^{2}=0.5$} & 50 & 0.771 & 0.663 & 0.734 & 0.762 & 0.528 & 0.257 & 0.440 & 0.501 \\
\hline & 75 & 0.748 & 0.637 & 0.711 & 0.735 & 0.528 & 0.223 & 0.435 & 0.487 \\
\hline & 100 & 0.739 & 0.618 & 0.700 & 0.727 & 0.524 & 0.207 & 0.411 & 0.469 \\
\hline & 150 & 0.714 & 0.594 & 0.671 & 0.696 & 0.522 & 0.189 & 0.404 & 0.468 \\
\hline & 200 & 0.702 & 0.569 & 0.654 & 0.686 & 0.511 & 0.180 & 0.389 & 0.463 \\
\hline \multirow[t]{5}{*}{$R^{2}=0.75$} & 50 & 0.968 & 0.882 & 0.953 & 0.965 & 0.918 & 0.149 & 0.801 & 0.885 \\
\hline & 75 & 0.966 & 0.878 & 0.950 & 0.962 & 0.925 & 0.121 & 0.801 & 0.895 \\
\hline & 100 & 0.959 & 0.865 & 0.941 & 0.953 & 0.925 & 0.108 & 0.803 & 0.895 \\
\hline & 150 & 0.960 & 0.853 & 0.939 & 0.955 & 0.934 & 0.100 & 0.808 & 0.899 \\
\hline & 200 & 0.958 & 0.846 & 0.935 & 0.953 & 0.938 & 0.095 & 0.813 & 0.910 \\
\hline
\end{tabular}

See notes to Table 4.

Table D.2: Small-sample power, further $c$

\begin{tabular}{|c|c|c|c|c|c|c|c|c|c|}
\hline DGP & $T$ & $\hat{F}$ & $t_{\gamma}^{\mathrm{ECR}}$ & $\tilde{\chi}_{\mathcal{I}}^{2}$ & $U R_{\psi_{\mathcal{I}}}$ & $\lambda_{\max }$ & $t_{\gamma}^{\mathrm{ADF}}$ & $\tilde{\chi}_{\mathcal{I}}^{2}$ & $U R_{\psi_{\mathcal{I}}}$ \\
\hline \multirow[t]{5}{*}{ (A) } & 50 & 0.303 & 0.265 & 0.285 & 0.293 & 0.144 & 0.186 & 0.171 & 0.196 \\
\hline & 75 & 0.264 & 0.231 & 0.249 & 0.258 & 0.141 & 0.163 & 0.153 & 0.171 \\
\hline & 100 & 0.247 & 0.214 & 0.234 & 0.241 & 0.133 & 0.147 & 0.140 & 0.161 \\
\hline & 150 & 0.232 & 0.202 & 0.224 & 0.223 & 0.133 & 0.140 & 0.136 & 0.152 \\
\hline & 200 & 0.219 & 0.190 & 0.203 & 0.210 & 0.131 & 0.128 & 0.129 & 0.148 \\
\hline \multirow[t]{5}{*}{ (C) } & 50 & 0.175 & 0.184 & 0.183 & 0.179 & 0.107 & 0.197 & 0.157 & 0.179 \\
\hline & 75 & 0.161 & 0.165 & 0.166 & 0.164 & 0.103 & 0.175 & 0.138 & 0.164 \\
\hline & 100 & 0.153 & 0.162 & 0.161 & 0.155 & 0.098 & 0.170 & 0.135 & 0.155 \\
\hline & 150 & 0.146 & 0.154 & 0.150 & 0.147 & 0.097 & 0.159 & 0.130 & 0.147 \\
\hline & 200 & 0.131 & 0.142 & 0.138 & 0.135 & 0.098 & 0.143 & 0.119 & 0.135 \\
\hline
\end{tabular}

See notes to Table 4. For $\operatorname{DGP}(\mathrm{A}), R^{2}=0.25$ and for $(\mathrm{A})$ and $(\mathrm{C}), c=-10$. 
Table D.3: Small-sample power, further $c$

\begin{tabular}{|c|c|c|c|c|c|c|c|c|c|}
\hline DGP & $T$ & $\hat{\hat{F}}$ & $t_{\gamma}^{\mathrm{ECR}}$ & $\tilde{\chi}_{\mathcal{I}}^{2}$ & $U R_{\psi_{\mathcal{I}}}$ & $\lambda_{\max }$ & $t_{\gamma}^{\mathrm{ADF}}$ & $\tilde{\chi}_{\mathcal{I}}^{2}$ & $U R_{\psi_{\mathcal{I}}}$ \\
\hline \multirow[t]{5}{*}{ (A) } & 50 & 0.806 & 0.788 & 0.804 & 0.805 & 0.537 & 0.602 & 0.641 & 0.626 \\
\hline & 75 & 0.782 & 0.763 & 0.784 & 0.777 & 0.528 & 0.544 & 0.609 & 0.597 \\
\hline & 100 & 0.743 & 0.720 & 0.741 & 0.738 & 0.506 & 0.485 & 0.560 & 0.543 \\
\hline & 150 & 0.729 & 0.701 & 0.726 & 0.724 & 0.497 & 0.461 & 0.544 & 0.534 \\
\hline & 200 & 0.710 & 0.688 & 0.710 & 0.705 & 0.487 & 0.445 & 0.531 & 0.514 \\
\hline \multirow[t]{5}{*}{ (C) } & 50 & 0.465 & 0.493 & 0.484 & 0.474 & 0.346 & 0.600 & 0.530 & 0.533 \\
\hline & 75 & 0.440 & 0.470 & 0.458 & 0.451 & 0.334 & 0.556 & 0.496 & 0.501 \\
\hline & 100 & 0.407 & 0.437 & 0.429 & 0.418 & 0.306 & 0.513 & 0.458 & 0.446 \\
\hline & 150 & 0.388 & 0.420 & 0.409 & 0.398 & 0.298 & 0.481 & 0.430 & 0.421 \\
\hline & 200 & 0.370 & 0.409 & 0.395 & 0.384 & 0.294 & 0.466 & 0.424 & 0.409 \\
\hline
\end{tabular}

See notes to Table 4. For DGP(A), $R^{2}=0.25$ and for $(\mathrm{A})$ and $(\mathrm{C}), c=-20$.

\section{Appendix E Additional Empirical Results}

Table E.1: Frequencies of test results in applied studies and the combination tests: combining $\lambda_{\max }$ and $t_{\gamma}^{\mathrm{ADF}}$

number of cases in which...

\begin{tabular}{|c|c|c|c|c|c|c|c|c|}
\hline & \multicolumn{3}{|c|}{...single test results... } & & \multicolumn{4}{|c|}{$\begin{array}{l}\text {...in case of conflicting } \\
\text { results: 'preferred' test }{ }^{\dagger}\end{array}$} \\
\hline & $r$ & $\neg r$ & & $\sum$ & & $r$ & $\neg r$ & $\sum$ \\
\hline$\tilde{\chi}_{\mathcal{I}}^{2}(2): r$ & 64 & 0 & 30 & 94 & $\tilde{\chi}_{\mathcal{I}}^{2}(2): r$ & 15 & 10 & 25 \\
\hline$\tilde{\chi}_{\mathcal{I}}^{2}(2): \neg r$ & 0 & 52 & 13 & 65 & $\tilde{\chi}_{\mathcal{I}}^{2}(2): \neg r$ & 8 & 5 & 13 \\
\hline$\sum$ & 64 & 52 & 43 & 159 & $\sum$ & 23 & 15 & 38 \\
\hline $\begin{array}{l}\tilde{\chi}_{\mathcal{I}}^{2}(2) \text { abl } \\
r: \text { test re } \\
\dagger: \text { Test ty } \\
\text { Absolute } \\
(2004) . \text { S } \\
\text { The } \tilde{\chi}_{\mathcal{I}}^{2}(2\end{array}$ & $\begin{array}{l}\text { on } \\
\text { ou } \\
\text { le t }\end{array}$ & $\begin{array}{l}\chi_{\mathcal{I}} \\
\text { whic } \\
\text { acies } \\
\text { sts in }\end{array}$ & $\begin{array}{l}\left.t_{\gamma}^{\mathrm{ADF}}\right) . \\
\text { es not } \mathrm{r} \\
\text { lusions } \\
\text { integrat } \\
\text { Engle a }\end{array}$ & $\begin{array}{l}\text { ct } \\
\text { the } \\
\text {-test } \\
\text { Gra }\end{array}$ & $\begin{array}{l}\text { al study we } \\
\text { lts for data } \\
\text { (1987) and } \\
\text { Section } 3 .\end{array}$ & $\begin{array}{l}\text { bas } \\
\text { from } \\
\text { han }\end{array}$ & d (se & $\begin{array}{l}\text { 17). } \\
\text { et al. } \\
\text { tests. }\end{array}$ \\
\hline
\end{tabular}

